ASC Report No. 18/2012

The structure of a set of positive solutions to Dirichlet BVPs with time and space singularities

I. Rachunková, A. Spielauer, S. Staněk and E.B. Weinmüller 


\section{Most recent ASC Reports}

17/2012 J.-F. Mennemann, A. Jüngel, and H. Kosina

Transient Schrödinger-Poisson simulations of a high-frequency resonant tunneling diode oscillator

16/2012 N. Zamponi and A. Jüngel

Two spinorial drift-diffusion models for quantum electron transport in graphene

15/2012 M. Aurada, M. Feischl, T. Führer, M. Karkulik, D. Praetorius

Efficiency and optimality of some weighted-residual error estimator for adaptive 2D boundary element methods

14/2012 I. Higueras, N. Happenhofer, O. Koch, and F. Kupka

Optimized Imex Runge-Kutta methods for simulations in astrophysics: A detailed study

13/2012 H. Woracek

Asymptotics of eigenvalues for a class of singular Krein strings

12/2012 H. Winkler, H. Woracek

A growth condition for Hamiltonian systems related with Krein strings

11/2012 B. Schörkhuber, T. Meurer, and A. Jüngel

Flatness-based trajectory planning for semilinear parabolic PDEs

10/2012 M. Karkulik, D. Pavlicek, and D. Praetorius

On 2D newest vertex bisection: Optimality of mesh-closure and $H^{1}$-stability of $L_{2}$-projection

09/2012 J. Schöberl and C. Lehrenfeld

Domain Decomposition Preconditioning for High Order Hybrid Discontinuous Galerkin Methods on Tetrahedral Meshes

08/2012 M. Aurada, M. Feischl, T. Führer, M. Karkulik, J.M. Melenk, D. Praetorius Classical FEM-BEM coupling methods: nonlinearities, well-posedness, and adaptivity

Institute for Analysis and Scientific Computing

Vienna University of Technology

Wiedner Hauptstraße 8-10

1040 Wien, Austria

E-Mail: admin@asc.tuwien.ac.at

WWW: http://www.asc.tuwien.ac.at

FAX: $\quad+43-1-58801-10196$

ISBN 978-3-902627-05-6

(c) Alle Rechte vorbehalten. Nachdruck nur mit Genehmigung des Autors.

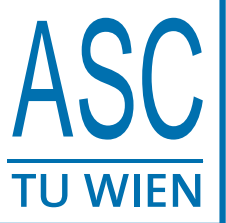




\title{
The structure of a set of positive solutions to Dirichlet BVPs with time and space singularities
}

\author{
Irena Rachůnkováa ${ }^{a}, \quad$ Alexander Spielauer ${ }^{b}$, Svatoslav Staněk $^{a}$ \\ and Ewa B. Weinmüller ${ }^{b}$ \\ ${ }^{a}$ Department of Mathematical Analysis, Faculty of Science, \\ Palacký University, 17. listopadu 12, CZ-771 46 Olomouc, Czech Republic \\ e-mail: irena.rachunkova@upol.cz svatoslav.stanek@upol.cz \\ ${ }^{b}$ Department of Analysis and Scientific Computing, Vienna University of Technology, \\ Wiedner Hauptstraße 8-10, A-1040 Wien, Austria \\ e-mail: alexander.spielauer@gmx.net e.weinmueller@tuwien.ac.at
}

\begin{abstract}
The paper discusses the solvability of the singular Dirichlet boundary value problem

$$
u^{\prime \prime}(t)+\frac{a}{t} u^{\prime}(t)-\frac{a}{t^{2}} u(t)=f\left(t, u(t), u^{\prime}(t)\right), \quad u(0)=0, u(T)=0 .
$$

Here $a \in(-\infty,-1)$ and $f$ satisfies the local Carathéodory conditions on $[0, T] \times \mathcal{D}$, where $\mathcal{D}=(0, \infty) \times \mathbb{R}$. It is shown that the cardinality of the set $\mathcal{L}$ of all positive solutions to the problem is a continuum. In addition, the structure and the properties of the set $\mathcal{L}$ are described. Applications and numerical simulations of the results are presented.
\end{abstract}

Mathematics Subject Classification 2010: 34B18, 34B16, 34A12

Key words: nonlinear ordinary differential equation of the second order, time and space singularities, set of all positive solutions, Leray-Schauder nonlinear alternative.

\section{Introduction}

We consider the singular Dirichlet boundary value problem

$$
\begin{aligned}
& u^{\prime \prime}(t)+\frac{a}{t} u^{\prime}(t)-\frac{a}{t^{2}} u(t)=f\left(t, u(t), u^{\prime}(t)\right), \\
& u(0)=0, \quad u(T)=0,
\end{aligned}
$$


where $a \in(-\infty,-1)$. Here, $f$ satisfies the local Carathéodory conditions on $[0, T] \times \mathcal{D}$, where $\mathcal{D}=(0, \infty) \times \mathbb{R}$.

We recall that a function $h:[0, T] \times \mathcal{A} \rightarrow \mathbb{R}, \mathcal{A} \subset \mathbb{R} \times \mathbb{R}$, satisfies the local Carathéodory conditions on $[0, T] \times \mathcal{A}$, if

(i) $h(\cdot, x, y):[0, T] \rightarrow \mathbb{R}$ is measurable for all $(x, y) \in \mathcal{A}$,

(ii) $h(t, \cdot, \cdot): \mathcal{A} \rightarrow \mathbb{R}$ is continuous for a.e. $t \in[0, T]$,

(iii) for each compact set $\mathcal{U} \subset \mathcal{A}$ there exists a function $m_{\mathcal{U}} \in L^{1}[0, T]$ such that

$$
|h(t, x, y)| \leq m_{\mathcal{U}}(t) \text { for a.e. } t \in[0, T] \text { and all }(x, y) \in \mathcal{U} \text {. }
$$

For such functions we use the notation $h \in \operatorname{Car}([0, T] \times \mathcal{A})$.

We see that $(0, y) \notin \mathcal{D}$ for each $y \in \mathbb{R}$, and hence $f(t, x, y)$ may be singular (unbounded in our case) at $x=0$. Equation (1.1a) has a time singularity at $t=0$ due to the structure of the differential operator on its left hand side. This operator has the equivalent form $\left(t^{-a}\left(t^{a} u\right)^{\prime}\right)^{\prime}$ and, after the substitution $v(t)=t^{a} u(t)$ it takes the form $\left(t^{-a} v^{\prime}(t)\right)^{\prime}$. Therefore, results derived for equation (1.1a) also apply for the modified equation $\left(t^{-a} v^{\prime}(t)\right)^{\prime}=g\left(t, v(t), v^{\prime}(t)\right)$. Such type of models arises in the study of phase transitions of Van der Waals fluids [3], [8], [12], [14], [18], in population genetics, in models for the spatial distribution of the genetic composition of a population [6], [7], in the homogenenous nucleation theory [1], in relativistic cosmology in description of particles which can be treated as domains in the universe [15], and in the nonlinear field theory [9], in particular, when describing bubbles generated by scalar fields of the Higgs type in the Minkowski spaces [5].

Problem (1.1), where $f$ has no singularity at $x=0$, i.e. $f$ satisfies the local Carathéodory conditions on $[0, T] \times \overline{\mathcal{D}}$, where $\overline{\mathcal{D}}=[0, \infty) \times \mathbb{R}$, has been investigated in [16]. This paper provides a comprehensive study of the set of all positive solutions of problem (1.1).

Systems of the form

$$
\begin{aligned}
& u^{\prime \prime}(t)-\frac{A_{1}}{t} u^{\prime}(t)-\frac{A_{0}}{t^{2}} u(t)=f\left(t, u(t), u^{\prime}(t)\right), \quad t \in(0, T], \\
& G\left(u(0), u^{\prime}(0), u(1), u^{\prime}(1)\right)=0, \quad u \in C^{1}[0,1],
\end{aligned}
$$

where, $A_{1}$ and $A_{0}$ are real valued $n \times n$ matrices, $f:(0,1] \times \mathbb{R}^{n} \times \mathbb{R}^{n} \rightarrow \mathbb{R}^{n}$ and $G: \mathbb{R}^{n} \times \mathbb{R}^{n} \times \mathbb{R}^{n} \times \mathbb{R}^{n} \rightarrow \mathbb{R}^{m}$ are smooth functions, $m \leq 2 n$, have been studied in [19]. The missing $2 n-m$ conditions have to be formulated in such a way that the requirement $u \in C^{1}[0,1]$ is satisfied. The main aim in [19] was to investigate the structure of boundary conditions which yield a well-posed boundary value problem. Moreover, in linear case, the existence and uniqueness theory was provided and the smoothness of $u$ was studied. In the nonlinear case, sufficient conditions for $u$ to be isolated, or locally unique, have been specified.

The approach taken in [19] is based on a technique developed in [10]. Instead of investigating directly the second order system (1.2a), its first order 
form obtained after the so called Euler transformation $y(t)=\left(y_{1}(t), y_{2}(t)\right)^{T}:=$ $\left(u(t), t u^{\prime}(t)^{T}\right.$ is analyzed,

$$
y^{\prime}(t)-\frac{M}{t} y(t)=F(t, y(t)), \quad t \in(0, T]
$$

where

$$
M=\left(\begin{array}{cc}
0 & I \\
A_{0} & A_{1}+I
\end{array}\right), \quad F(t, y(t))=\left(\begin{array}{c}
0 \\
t f\left(t, y_{1}(t), \frac{y_{2}(t)}{t}\right)
\end{array}\right) .
$$

It turns out that the eigenvalues of $M$ play crucial role in describing the solution structure and therefore, the structure of boundary conditions necessary for the solution to be continuous on $[0,1]$. This is clear, because the fundamental matrix solution reads $Y(t)=e^{M \ln t}$.

In case of the homogeneous differential equation (1.1a), we have

$$
y^{\prime}(t)-\frac{M}{t} y(t)=0, \quad t \in(0, T], \quad M=\left(\begin{array}{cc}
0 & 1 \\
a & -a+1
\end{array}\right),
$$

and the eigenvalues of $M$ are $\lambda_{1}=-a$ and $\lambda_{2}=1$. By decoupling (1.4), we conclude that the general solution of the homogeneous problem (1.1a) is $u(t)=$ $c_{1} t^{\lambda_{1}}+c_{2} t^{\lambda_{2}}=c_{1} t^{-a}+c_{2} t$ with arbitrary constants ${ }^{1} c_{1}, c_{2} \in \mathbb{R}$. Since both eigenvalues are positive, it follows immediately from [19] that the problem (1.1) is not well-posed and has infinitely many solutions. By prescribing finial conditions, $u(T)=0, u^{\prime}(T)=-c$, instead of (1.1b), the problem becomes well-posed and can be solved numerically, cf. Section 6 . Since we are interested in positive solutions, we choose $c \geq 0$.

The aim of this paper is to extend results from [16] and [19] to problem (1.1) having space singularities. We discuss its solvability and describe the structure of the set $\mathcal{L}$ of all its positive solutions. The existence results are proved by the combination of regularization and sequential techniques with the Leray-Schauder nonlinear alternative. We also show the interesting result stating that for each $c \geq 0$ there exists a function $u \in \mathcal{L}$ such that $u^{\prime}(T)=-c$, and hence, the cardinality of the set $\mathcal{L}$ is a continuum. Finally, by means of three nonlinear test examples, we illustrate the theoretical findings. These examples are solved using a MATLAB code bvpsuite [13] based on collocation.

We start by introducing the necessary notions.

\footnotetext{
${ }^{1}$ Note, that we obtain the same solution if in

$$
u^{\prime \prime}(t)+\frac{a}{t} u^{\prime}(t)-\frac{a}{t^{2}} u(t)=0
$$

the substitution $u(t):=t^{\lambda}$ is made. Clearly, in the scalar case, the roots of the so called characteristic polynomial $\lambda(\lambda-1)+a \lambda-a=0$ coincide with the eigenvalues of $M$.
} 
Let us denote by $L^{1}[0, T]$ the set of functions which are Lebesgue integrable on $[0, T]$ equipped with the norm $\|x\|_{1}=\int_{0}^{T}|x(t)| \mathrm{d} t$. Moreover, let us denote by $C[0, T]$ and $C^{1}[0, T]$ the set of functions being continuous on $[0, T]$, and having continuous first derivative on $[0, T]$, respectively. The norm on $C[0, T]$ and $C^{1}[0, T]$ is defined as $\|x\|_{\infty}=\max _{t \in[0, T]}|x(t)|$ and $\|x\|_{C^{1}}=\|x\|_{\infty}+\left\|x^{\prime}\right\|_{\infty}$, respectively. Finally, we denote by $A C^{1}[0, T]$ the set of functions which have absolutely continuous first derivatives on $[0, T]$, while $A C_{l o c}^{1}(0, T]$ is the set of functions having absolutely continuous derivatives on each compact subinterval of $(0, T]$.

We say that $u:[0, T] \rightarrow \mathbb{R}$ is a positive solution of problem (1.1) if $u \in$ $A C^{1}[0, T], u>0$ on $(0, T), u$ satisfies the boundary conditions (1.1b) and (1.1a) holds for a.e. $t \in[0, T]$.

We work with the following conditions on $f$ in (1.1a).

$\left(H_{1}\right) \quad f \in \operatorname{Car}([0, T] \times \mathcal{D})$, where $\mathcal{D}=(0, \infty) \times \mathbb{R}$.

$\left(H_{2}\right)$ There exists $\Delta>0$ such that

$$
\Delta \leq f(t, x, y) \text { for a.e. } t \in[0, T] \text { and all }(x, y) \in \mathcal{D} \text {. }
$$

$\left(H_{3}\right)$ For a.e. $t \in[0, T]$ and all $(x, y) \in \mathcal{D}$ the estimate

$$
f(t, x, y) \leq h(t, x,|y|)+g(x)
$$

holds, where $h \in \operatorname{Car}([0, T] \times \mathcal{A}), \mathcal{A}=[0, \infty) \times[0, \infty)$ and $g \in C(0, \infty)$ are positive, $h(t, x, y)$ is nondecreasing in the variables $x, y, g$ is nonincreasing, and

$$
\lim _{x \rightarrow \infty} \frac{1}{x} \int_{0}^{T} h(t, x, x) \mathrm{d} t=0, \quad \int_{0}^{1} g\left(s^{2}\right) \mathrm{d} s<\infty .
$$

Remark 1.1 Let $g$ satisfy the conditions given in $\left(H_{3}\right)$. Then $\int_{0}^{b} g\left(c s^{2}\right) \mathrm{d} s<\infty$ for each $b, c \in(0, \infty)$, and it follows from the inequality

$$
t(T-t)^{2} \geq \begin{cases}\frac{T}{2} t^{2}, & t \in\left[0, \frac{T}{2}\right] \\ \frac{T}{2}(T-t)^{2}, & t \in\left[\frac{T}{2}, T\right]\end{cases}
$$

that

$$
\int_{0}^{T} g\left(c t(T-t)^{2}\right) \mathrm{d} t<\infty \text { for each } c \in(0, \infty)
$$


The paper is organized as follows. Section 2 contains inequalities which we will require in the next three sections. Section 3 is devoted to the study of limit properties as $t \rightarrow 0+$ of solutions to equations of the following type:

$$
u^{\prime \prime}(t)+\frac{-}{t} u^{\prime}(t)-\frac{a}{t^{2}} u(t)=r\left(t, u(t), u^{\prime}(t)\right),
$$

where the function $r$ satisfies the global Carathéodory conditions on $[0, T] \times$ $\mathbb{R}^{2}$. In Section 4, we investigate auxiliary regular problems associated with the singular problem (1.1). We show their solvability and properties of their solutions. Existence results for singular problem (1.1) are given in Section 5. Here, in addition, the properties of the set $\mathcal{L}$ of all positive solutions to the problem are derived together with some applications. Finally, in Section 6, we illustrate the theoretical findings by means of numerical experiments.

Throughout the paper $a \in(-\infty,-1)$.

\section{Preliminaries}

This section contains inequalities required for the proofs in Sections 3 to 5 .

Lemma 2.1 Let $p \in L^{1}[0, T]$. Then the inequalities

$$
\begin{gathered}
\left|t^{-a-1} \int_{t}^{T} s^{a+1} p(s) \mathrm{d} s\right| \leq \int_{t}^{T}|p(s)| \mathrm{d} s \\
\left|\int_{t}^{T} s^{-a-2}\left(\int_{s}^{T} \xi^{a+1} p(\xi) \mathrm{d} \xi\right) \mathrm{d} s\right| \leq \frac{1}{|a+1|} \int_{t}^{T}|p(s)| \mathrm{d} s
\end{gathered}
$$

hold for $t \in[0, T]$.

Proof. See [16, Lemma 1].

Lemma 2.2 The inequality

$$
\int_{t}^{T} s^{-a-2}\left(\int_{s}^{T} \xi^{a+1} \mathrm{~d} \xi\right) \mathrm{d} s \geq \begin{cases}\frac{(T-t)^{2}}{2 T}, & a \in[-3,-1) \\ \frac{(T-t)^{2}}{2 T(a+2)^{2}}, & a \in(-\infty,-3)\end{cases}
$$

holds for $t \in[0, T]$. 
Proof. Let $a \in[-2,-1)$, then

$$
\begin{aligned}
\int_{t}^{T} s^{-a-2}\left(\int_{s}^{T} \xi^{a+1} \mathrm{~d} \xi\right) \mathrm{d} s & \geq T^{a+1} \int_{t}^{T}(T-s) s^{-a-2} \mathrm{~d} s \\
& \geq \frac{1}{T} \int_{t}^{T}(T-s) \mathrm{d} s=\frac{(T-t)^{2}}{2 T}
\end{aligned}
$$

In particular,

$$
\int_{t}^{T} s^{-a-2}\left(\int_{s}^{T} \xi^{a+1} \mathrm{~d} \xi\right) \mathrm{d} s \geq \frac{(T-t)^{2}}{2 T} \text { for } t \in[0, T] \text { and } a \in[-2,-1) .
$$

Let $a \in(-\infty,-2)$. Then

$$
\begin{aligned}
\int_{t}^{T} s^{-a-2}\left(\int_{s}^{T} \xi^{a+1} \mathrm{~d} \xi\right) \mathrm{d} s= & \frac{1}{|a+2|} \int_{t}^{T}\left(1-\left(\frac{s}{T}\right)^{-a-2}\right) \mathrm{d} s \\
& =\frac{T}{|a+2|} \int_{t / T}^{1}(1-s)^{-a-2} \mathrm{~d} s
\end{aligned}
$$

for $t \in[0, T]$. Choose $p(x):=1-x^{\beta}-\beta(1-x)$ for $x \in[0,1]$, where $\beta \in(0,1)$. Then $p(0)=1-\beta>0, p(1)=0$, and since $p^{\prime}(x)=\beta\left(1-x^{\beta-1}\right)<0$ for $x \in(0,1)$, we have $p>0$ on $[0,1)$. Consequently, $1-x^{\beta} \geq \beta(1-x)$ for $x \in[0,1]$ and $\beta \in(0,1]$. This gives for $\beta=-a-2$,

$$
1-x^{-a-2} \geq|a+2|(1-x) \text { for } x \in[0,1] \text { and } a \in[-3,-2) .
$$

Hence, by (2.5), the relation

$$
\int_{t}^{T} s^{-a-2}\left(\int_{s}^{T} \xi^{a+1} \mathrm{~d} \xi\right) \mathrm{d} s \geq T \int_{t / T}^{1}(1-s) \mathrm{d} s=\frac{(T-t)^{2}}{2 T}
$$

is satisfied for $t \in[0,1]$ and $a \in[-3,-2)$.

In order to verify $(2.3)$ for $a \in(-\infty,-3)$, let

$$
r(x):=1-x^{\gamma}-\frac{1-x}{\gamma} \text { for } x \in[0,1]
$$

where $\gamma>1$. Then $r(0)=1-\frac{1}{\gamma}>0, r(1)=0, r^{\prime}(x)=-\gamma x^{\gamma-1}+\frac{1}{\gamma}$, and $r^{\prime \prime}(x)=-\gamma(\gamma-1) x^{\gamma-2}$. Hence, $r^{\prime \prime}<0$ on $(0,1]$, and since $r^{\prime}(0)=\frac{1}{\gamma}>0$ and $r^{\prime}(1)=-\gamma+\frac{1}{\gamma}<0$, we conclude that $r \geq 0$ on $[0,1]$. That is $1-x^{\gamma} \geq \frac{1-x}{\gamma}$ for $x \in[0,1]$ and $\gamma>1$. Therefore, for $\gamma=-a-2$,

$$
1-x^{-a-2} \geq \frac{1-x}{|a+2|} \text { for } x \in[0,1] \text { and } a \in(-\infty,-3),
$$


and so, by (2.5),

$$
\int_{t}^{T} s^{-a-2}\left(\int_{s}^{T} \xi^{a+1} \mathrm{~d} \xi\right) \mathrm{d} s \geq \frac{T}{(a+2)^{2}} \int_{t / T}^{1}(1-s) \mathrm{d} s=\frac{(T-t)^{2}}{2 T(a+2)^{2}},
$$

for $t \in[0, T]$ and $a \in(-\infty,-3)$.

Inequality (2.3) now follows from (2.4), (2.6) and (2.7).

\section{$3 \quad$ Limit properties of solutions}

In this section we consider the differential equation

$$
u^{\prime \prime}(t)+\frac{a}{t} u^{\prime}(t)-\frac{a}{t^{2}} u(t)=r\left(t, u(t), u^{\prime}(t)\right), \quad t \in(0, T],
$$

where $r$ satisfies the global Carathéodory condition on $[0, T] \times \mathbb{R}^{2}$, that is,

$\left(H_{4}\right) r(\cdot, x, y):[0, T] \rightarrow \mathbb{R}$ is measurable for all $(x, y) \in \mathbb{R} \times \mathbb{R}, r(t, \cdot, \cdot): \mathbb{R} \times \mathbb{R} \rightarrow$ $\mathbb{R}$ is continuous for a.e. $t \in[0, T]$, and there exists $\mu \in L^{1}[0, T]$ such that

$$
|r(t, x, y)| \leq \mu(t) \text { for a.e. } t \in[0, T] \text { and all }(x, y) \in \mathbb{R}^{2} .
$$

We now describe the analytical form and the asymptotic behavior for $t \rightarrow 0+$ of functions $u$ satisfying (3.1) a.e. on $[0, T]$.

Lemma 3.1 Let condition $\left(H_{4}\right)$ hold. Let the function $u \in A C_{l o c}^{1}(0, T]$ satisfy (3.1) for a.e. $t \in[0, T]$. Then $u$ can be extended on $[0, T]$ with $u \in A C^{1}[0, T]$ and the representation

$$
u(t)=c_{1} t+c_{2} t^{-a}+t \int_{t}^{T} s^{-a-2}\left(\int_{s}^{T} \xi^{a+1} r\left(\xi, u(\xi), u^{\prime}(\xi)\right) \mathrm{d} \xi\right) \mathrm{d} s
$$

where $c_{1}, c_{2} \in \mathbb{R}$, holds for $t \in[0, T]$.

Proof. Keeping in mind that $u$ is fixed, consider the Euler linear differential equation

$$
v^{\prime \prime}(t)+\frac{a}{t} v^{\prime}(t)-\frac{a}{t^{2}} v(t)=r\left(t, u(t), u^{\prime}(t)\right) .
$$

Each function $v \in A C_{l o c}^{1}(0, T]$ satisfying (3.4) a.e. on $[0, T]$ has the form

$$
v(t)=c_{1}^{*} t+c_{2}^{*} t^{-a}+t \int_{t}^{T} s^{-a-2}\left(\int_{s}^{T} \xi^{a+1} r\left(\xi, u(\xi), u^{\prime}(\xi)\right) \mathrm{d} \xi\right) \mathrm{d} s,
$$


where $c_{1}^{*}, c_{2}^{*} \in \mathbb{R}$. Since, by the assumption $u \in A C_{l o c}^{1}(0, T]$ satisfies (3.4) for a.e. $t \in[0, T]$, there exists $c_{1}, c_{2} \in \mathbb{R}$ such that equality (3.3) holds for $t \in(0, T]$. In order to prove that $u$ can be extended on $[0, T]$ as a function in $A C^{1}[0, T]$, and consequently, that (3.3) is satisfied for $t \in[0, T]$, we have to show that

$$
\int_{0}^{T}\left|u^{\prime \prime}(t)\right| \mathrm{d} s<\infty
$$

By (3.3),

$\frac{a}{t} u^{\prime}(t)-\frac{a}{t^{2}} u(t)=-a t^{-a-2}\left(c_{2}(a+1)+\int_{t}^{T} s^{a+1} r\left(s, u(s), u^{\prime}(s)\right) \mathrm{d} s\right)$ for $t \in(0, T]$,

and then using (3.2) we obtain

$$
\left|\frac{a}{t} u^{\prime}(t)-\frac{a}{t^{2}} u(t)\right| \leq|a| t^{-a-2}\left(\left|c_{2}(a+1)\right|+\int_{t}^{T} s^{a+1} \mu(s) \mathrm{d} s\right) \text { for } t \in(0, T] .
$$

Hence, by (2.2),

$$
\begin{aligned}
\int_{0}^{T}\left|u^{\prime \prime}(t)\right| \mathrm{d} s & \leq \int_{0}^{T}\left|\frac{a}{t} u^{\prime}(t)-\frac{a}{t^{2}} u(t)\right| \mathrm{d} t+\int_{0}^{T}\left|r\left(t, u(t), u^{\prime}(t)\right)\right| \mathrm{d} t \\
\leq & |a|\left(\left|c_{2}(a+1)\right| \int_{0}^{T} t^{-a-2} \mathrm{~d} t+\int_{0}^{T} t^{-a-2}\left(\int_{t}^{T} s^{a+1} \mu(s) \mathrm{d} s\right) \mathrm{d} t\right) \\
& \quad+\int_{0}^{T} \mu(s) \mathrm{d} s \\
\leq & \left|a c_{2}\right| T^{-a-1}+\frac{(2 a+1)\|\mu\|_{1}}{a+1} .
\end{aligned}
$$

Consequently, (3.5) holds and this completes the proof.

The following corollaries extend the statement of Lemma 3.1 for $r \in \operatorname{Car}([0, T] \times$ $\mathbb{R}^{2}$ ), that is for $r$ satisfying only the local Carathéodory conditions on $[0, T] \times \mathbb{R}^{2}$.

Corollary 3.2 Let $r \in \operatorname{Car}\left([0, T] \times \mathbb{R}^{2}\right)$ and let $u \in A C_{l o c}^{1}(0, T]$ satisfy (3.1) a.e. on $[0, T]$. Assume also that

$$
L:=\sup \left\{|u(t)|+\left|u^{\prime}(t)\right|: t \in(0, T]\right\}<\infty
$$

holds. Then, the assertion of Lemma 3.1 is satisfied.

Proof. Let

$$
\rho(z):= \begin{cases}L, & z>L \\ z, & |z| \leq L \\ -L, & z<-L\end{cases}
$$


and let $r^{*}(t, x, y):=r(t, \rho(x), \rho(y))$ for a.e. $t \in[0, T]$ and all $(x, y) \in \mathbb{R} \times \mathbb{R}$. Then $r^{*}$ satisfies the global Carathéodory conditions on $[0, T] \times \mathbb{R}^{2}$ and the equality

$$
u^{\prime \prime}(t)+\frac{a}{t} u^{\prime}(t)-\frac{a}{t^{2}} u(t)=r^{*}\left(t, u(t), u^{\prime}(t)\right)
$$

holds for a.e. $t \in[0, T]$. The result now follows from Lemma 3.1, where $r$ is replaced by $r^{*}$ in equation (3.1).

Corollary 3.3 Let $r \in \operatorname{Car}\left([0, T] \times \mathbb{R}^{2}\right)$ and let $u \in A C^{1}[0, T]$ be a solution of equation (3.1). Then there exist $c_{1}, c_{2} \in \mathbb{R}$ such that equality (3.3) is satisfied for $t \in[0, T]$.

Proof. We can apply Corollary 3.2, since $u \in A C^{1}[0, T]$ yields

$$
\sup \left\{|u(t)|+\left|u^{\prime}(t)\right|: t \in[0, T]\right\}<\infty .
$$

Remark 3.4 Corollary 3.3 shows that each solution $u \in A C^{1}[0, T]$ of equation (3.1) with $r \in \operatorname{Car}\left([0, T] \times \mathbb{R}^{2}\right)$ has the form given in (3.3), where $c_{1}, c_{2} \in \mathbb{R}$, and therefore, it satisfies $u(0)=0$. Consequently, when discussing solutions $u \in A C^{1}[0, T]$ of equation (3.1) together with boundary conditions, especially including the condition $u(0)=u_{0}$, then, necessarily, $u_{0}=0$.

\section{Auxiliary regular problems}

Since equation (1.1a) is singular, we use the regularization and sequential techniques for solving problem (1.1). To this end, we define $f_{n}:[0, T] \times \mathbb{R}^{2} \rightarrow \mathbb{R}$, $n \in \mathbb{N}$, by the formula

$$
f_{n}(t, x, y)= \begin{cases}f(t, x, y), & x \geq \frac{1}{n} \\ f\left(t, \frac{1}{n}, y\right), & x<\frac{1}{n} .\end{cases}
$$

Under conditions $\left(H_{1}\right)-\left(H_{3}\right), f_{n} \in \operatorname{Car}\left([0, T] \times \mathbb{R}^{2}\right)$ and

$$
\left.\begin{array}{c}
\Delta \leq f_{n}(t, x, y) \text { for a.e. } t \in[0, T] \text { and all }(x, y) \in \mathbb{R}^{2}, \\
\qquad f_{n}(t, x, y) \leq h(t, 1+|x|,|y|)+g(|x|) \\
\text { for a.e. } t \in[0, T] \text { and all }(x, y) \in \mathbb{R}_{0} \times \mathbb{R} .
\end{array}\right\}
$$


Here $\mathbb{R}_{0}=\mathbb{R} \backslash\{0\}$. Hence,

$$
\left.\begin{array}{r}
\Delta \leq \lambda f_{n}(t, x, y)+(1-\lambda) \Delta \leq h(t, 1+|x|,|y|)+g(|x|) \\
\quad \text { for a.e. } t \in[0, T] \text { and all }(x, y) \in \mathbb{R}_{0} \times \mathbb{R}, \lambda \in[0,1] .
\end{array}\right\}
$$

We consider the differential equations

$$
u^{\prime \prime}(t)+\frac{a}{t} u^{\prime}(t)-\frac{a}{t^{2}} u(t)=f_{n}\left(t, u(t), u^{\prime}(t)\right), \quad n \in \mathbb{N},
$$

and

$$
u^{\prime \prime}(t)+\frac{a}{t} u^{\prime}(t)-\frac{a}{t^{2}} u(t)=\lambda f_{n}\left(t, u(t), u^{\prime}(t)\right)+(1-\lambda) \Delta, \quad \lambda \in[0,1], n \in \mathbb{N} .
$$

A function $u:[0, T] \rightarrow \mathbb{R}$ is called a solution of (4.4) if $u \in A C^{1}[0, T]$ and $u$ satisfies (4.4) for a.e. $t \in[0, T]$. Solutions of (4.5) are defined analogously.

Let us now define the boundary value problem (4.6) consisting of the differential equation specified in (4.4) subject to the boundary condition (1.1b),

$$
\begin{aligned}
& u^{\prime \prime}(t)+\frac{a}{t} u^{\prime}(t)-\frac{a}{t^{2}} u(t)=f_{n}\left(t, u(t), u^{\prime}(t)\right), \quad n \in \mathbb{N}, \\
& u(0)=0, \quad u(T)=0 .
\end{aligned}
$$

Lemma 4.1 Let condition $\left(H_{1}\right)$ hold. Then, all solutions $u \in A C^{1}[0, T]$ of problem (4.6) form a one-parameter system $\mathcal{A}$, where

$$
\begin{aligned}
\mathcal{A}= & \left\{c_{2} t\left(t^{-a-1}-T^{-a-1}\right)\right. \\
& \left.+t \int_{t}^{T} s^{-a-2}\left(\int_{s}^{T} \xi^{a+1} f_{n}\left(\xi, u(\xi), u^{\prime}(\xi)\right) \mathrm{d} \xi\right) \mathrm{d} s: c_{2} \in \mathbb{R}\right\} .
\end{aligned}
$$

Proof. Let $u \in A C^{1}[0, T]$ be a solution of problem (4.6). Since $u$ is a solution of (4.6a), the equality

$$
u(t)=c_{1} t+c_{2} t^{-a}+t \int_{t}^{T} s^{-a-2}\left(\int_{s}^{T} \xi^{a+1} f_{n}\left(\xi, u(\xi), u^{\prime}(\xi)\right) \mathrm{d} \xi\right) \mathrm{d} s
$$

holds for $t \in[0, T]$ by Corollary 3.3, where $c_{1}, c_{2} \in \mathbb{R}$. Then $u(0)=0$ and the condition $u(T)=0$ yields $c_{1}=-c_{2} T^{-a-1}$. Hence, by (4.7), $u \in \mathcal{A}$.

Let $u \in \mathcal{A}$, that is,

$$
u(t)=c_{2} t\left(t^{-a-1}-T^{-a-1}\right)+t \int_{t}^{T} s^{-a-2}\left(\int_{s}^{T} \xi^{a+1} f_{n}\left(\xi, u(\xi), u^{\prime}(\xi)\right) \mathrm{d} \xi\right) \mathrm{d} s
$$


for $t \in[0, T]$, where $c_{2} \in \mathbb{R}$. Then $u$ satisfies condition (4.6b) and

$$
\begin{gathered}
u^{\prime}(t)=-c_{2}\left(a t^{-a-1}+T^{-a-1}\right) \\
\quad+\int_{t}^{T} s^{-a-2}\left(\int_{s}^{T} \xi^{a+1} f_{n}\left(\xi, u(\xi), u^{\prime}(\xi)\right) \mathrm{d} \xi\right) \mathrm{d} s \\
\quad-t^{-a-1} \int_{t}^{T} s^{a+1} f_{n}\left(s, u(s), u^{\prime}(s)\right) \mathrm{d} s, \quad t \in[0, T], \\
u^{\prime \prime}(t)=a(a+1) c_{2} t^{-a-2}+a t^{-a-2} \int_{t}^{T} s^{a+1} f_{n}\left(s, u(s), u^{\prime}(s)\right) \mathrm{d} s \\
+f_{n}\left(t, u(t), u^{\prime}(t)\right), \text { for a.e. } t \in[0, T] .
\end{gathered}
$$

By $\left(H_{1}\right), f_{n}\left(t, u(t), u^{\prime}(t)\right) \in L^{1}[0, T]$ and consequently, (2.2) implies

$$
t^{-a-2} \int_{t}^{T} s^{a+1} f_{n}\left(s, u(s), u^{\prime}(s)\right) \mathrm{d} s \in L^{1}[0, T] .
$$

As a result, $u \in A C^{1}[0, T]$. Using (4.8), (4.9) and (4.10), we can verify that $u$ satisfies (4.6a) for a.e. $t \in[0, T]$.

In the following lemma, we discuss solutions $u$ of the boundary value problem:

$$
\begin{aligned}
& u^{\prime \prime}(t)+\frac{a}{t} u^{\prime}(t)-\frac{a}{t^{2}} u(t)=f_{n}\left(t, u(t), u^{\prime}(t)\right), \quad n \in \mathbb{N}, \\
& u(0)=0, \quad u(T)=0, \quad u^{\prime}(T)=-c, \quad c \geq 0 .
\end{aligned}
$$

In this problem $u$ satisfies, besides the Dirichlet conditions (4.6b), the additional condition

$$
u^{\prime}(T)=-c,
$$

for a fixed $c \geq 0$. Note that condition (4.12) together with (4.9) yields

$$
c_{2}=\frac{c}{a+1} T^{a+1} \leq 0
$$

in $(4.8)$.

Lemma 4.2 Let $\left(H_{1}\right)$ hold. Then a function $u \in A C^{1}[0, T]$ is a solution of problem (4.11) if and only if $u$ is a solution of the integral equation

$$
\begin{aligned}
u(t)= & t \frac{c T^{a+1}}{|a+1|}\left(T^{-a-1}-t^{-a-1}\right) \\
& +t \int_{t}^{T} s^{-a-2}\left(\int_{s}^{T} \xi^{a+1} f_{n}\left(\xi, u(\xi), u^{\prime}(\xi)\right) \mathrm{d} \xi\right) \mathrm{d} s
\end{aligned}
$$

in the set $C^{1}[0, T]$. 
Proof. $(\Rightarrow)$ Let us first assume that $u \in A C^{1}[0, T]$ is a solution of problem (4.11). Then $u \in \mathcal{A}$, that is $u$ satisfies (4.8), with $c_{2}$ given by (4.13). As a result, $u$ is a solution of (4.14) in $C^{1}[0, T]$.

$(\Leftarrow)$ Let now $u \in C^{1}[0, T]$ be a solution of (4.14). Then $u$ satisfies (4.8), (4.9) and (4.10) with $c_{2}$ given by (4.13). Therefore, $u$ satisfies boundary conditions (4.11b). The same reasoning as in the proof of Lemma 4.1 implies that $u \in$ $A C^{1}[0, T]$ and $u$ satisfies equation (4.11a) for a.e. $t \in[0, T]$.

Let

$$
M= \begin{cases}\frac{\Delta}{2 T}, & a \in[-3,-1), \\ \frac{\Delta}{2 T(a+2)^{2}}, & a \in(-\infty,-3),\end{cases}
$$

with $\Delta$ specified in condition $\left(H_{2}\right)$.

Consider a fixed $c \geq 0$. We now derive bounds for solutions of the boundary value problem

$$
\begin{aligned}
& u^{\prime \prime}(t)+\frac{a}{t} u^{\prime}(t)-\frac{a}{t^{2}} u(t)=\lambda f_{n}\left(t, u(t), u^{\prime}(t)\right)+(1-\lambda) \Delta, \quad \lambda \in[0,1](4.16 \mathrm{a}) \\
& u(0)=0, \quad u(T)=0, \quad u^{\prime}(T)=-\lambda c .
\end{aligned}
$$

Note, that this time $u$ satisfies additionally, besides the Dirichlet conditions (4.6b), the following condition

$$
u^{\prime}(T)=-\lambda c, \quad \lambda \in[0,1] .
$$

Lemma 4.3 Let conditions $\left(H_{1}\right)-\left(H_{3}\right)$ hold. Then, there exists a positive constant $S$ independent of $n$ and $\lambda$ such that for all solutions $u$ of problems (4.16) the estimates

$$
\begin{gathered}
u(t) \geq M t(T-t)^{2}, \quad t \in[0, t], \\
\|u\|_{\infty}<S T, \quad\left\|u^{\prime}\right\|_{\infty}<S,
\end{gathered}
$$

hold.

Proof. Let $u$ be a solution of problem (4.16) for some $n \in \mathbb{N}$ and $\lambda \in[0,1]$. Applying Lemma 4.2 to this problem we obtain the equality

$$
\begin{aligned}
u(t)= & \lambda t \frac{c T^{a+1}}{|a+1|}\left(T^{-a-1}-t^{-a-1}\right) \\
& +t \int_{t}^{T} s^{-a-2}\left(\int_{s}^{T} \xi^{a+1}\left[\lambda f_{n}\left(\xi, u(\xi), u^{\prime}(\xi)\right)+(1-\lambda) \Delta\right] \mathrm{d} \xi\right) \mathrm{d} s
\end{aligned}
$$


for $t \in[0, T]$. Since $c \geq 0, \lambda \geq 0$, we have due to (2.3), (4.1) and (4.15),

$$
u(t) \geq \Delta t \int_{t}^{T} s^{-a-2}\left(\int_{s}^{T} \xi^{a+1} \mathrm{~d} \xi\right) \mathrm{d} s \geq M t(T-t)^{2} \quad \text { for } t \in[0, T],
$$

and so (4.18) is true. Furthermore, $u>0$ on $(0, T)$ and $g(u(t)) \leq g\left(M t(T-t)^{2}\right)$ for $t \in(0, T)$ since $g$ is nonincreasing on $(0, \infty)$ by $\left(H_{3}\right)$. Hence,

$$
\|g(u(t))\|_{1} \leq\left\|g\left(M t(T-t)^{2}\right)\right\|_{1}=: W_{1}
$$

where $W_{1}<\infty$ by Remark 1.1. Note that the value of $W_{1}$ neither depends on the choice of solution $u$ to problem (4.16) nor on $n$ and $\lambda$. Since

$$
\begin{aligned}
u^{\prime}(t)= & \frac{\lambda c T^{a+1}}{|a+1|}\left(T^{-a-1}+a t^{-a-1}\right) \\
& +\int_{t}^{T} s^{-a-2}\left(\int_{s}^{T} \xi^{a+1}\left[\lambda f_{n}\left(\xi, u(\xi), u^{\prime}(\xi)\right)+(1-\lambda) \Delta\right] \mathrm{d} \xi\right) \mathrm{d} s \\
& \quad-t^{-a-1} \int_{t}^{T} s^{a+1}\left[\lambda f_{n}\left(s, u(s), u^{\prime}(s)\right)+(1-\lambda) \Delta\right] \mathrm{d} s, \quad t \in[0, T],
\end{aligned}
$$

it follows from $(2.1),(2.2),(4.3)$ and (4.21) that the relation

$$
\begin{aligned}
\left|u^{\prime}(t)\right| \leq c( & \left.\frac{1}{|a+1|}+1\right) \\
& +\int_{t}^{T} s^{-a-2}\left(\int_{s}^{T} \xi^{a+1}\left[h\left(\xi, 1+u(\xi),\left|u^{\prime}(\xi)\right|\right)+g(u(\xi))\right] \mathrm{d} \xi\right) \mathrm{d} s \\
& \quad+t^{-a-1} \int_{t}^{T} s^{a+1}\left[h\left(s, 1+u(s),\left|u^{\prime}(s)\right|\right)+g(u(s))\right] \mathrm{d} s \\
\leq & \left(\frac{1}{|a+1|}+1\right)\left(\int_{0}^{T} h\left(s, 1+u(s),\left|u^{\prime}(s)\right|\right) \mathrm{d} s+W_{1}+c\right) \\
\leq & \left(\frac{1}{|a+1|}+1\right)\left(\int_{0}^{T} h\left(s, 1+\|u\|_{\infty},\left\|u^{\prime}\right\|_{\infty}\right) \mathrm{d} s+W\right)
\end{aligned}
$$

is satisfied for $t \in[0, T]$ and $W:=W_{1}+c$.

In particular,

$$
\left\|u^{\prime}\right\|_{\infty} \leq\left(\frac{1}{|a+1|}+1\right)\left(\int_{0}^{T} h\left(s, 1+\|u\|_{\infty},\left\|u^{\prime}\right\|_{\infty}\right) \mathrm{d} s+W\right) .
$$

Since $u(t)=\int_{0}^{t} u^{\prime}(s) \mathrm{d} s$, we have

$$
\|u\|_{\infty} \leq T\left\|u^{\prime}\right\|_{\infty}
$$


and therefore

$$
\left\|u^{\prime}\right\|_{\infty} \leq\left(\frac{1}{|a+1|}+1\right)\left(\int_{0}^{T} h\left(s, 1+T\left\|u^{\prime}\right\|_{\infty},\left\|u^{\prime}\right\|_{\infty}\right) \mathrm{d} t+W\right) .
$$

By $\left(H_{3}\right), \lim _{x \rightarrow \infty} \frac{1}{x} \int_{0}^{T} h(t, 1+T x, x) \mathrm{d} t=0$, and consequently, there exists $S>0$ such that

$$
\left(\frac{1}{|a+1|}+1\right)\left(\int_{0}^{T} h(s, 1+T x, x) \mathrm{d} t+W\right)<x \text { for all } x \geq S .
$$

Now we conclude from the last relation and from (4.24) that $\left\|u^{\prime}\right\|_{\infty}<S$, and therefore, by (4.23), $\|u\|_{\infty}<S T$. Hence (4.19) holds and this completes the proof.

We are now in the position to show the existence of a solution of problem (4.11). This result is proved by the following nonlinear alternative of LeraySchauder type which follows, for example, from [2, Theorem 5.1].

Lemma 4.4 Let $X$ be a Banach space, $\Omega$ an open bounded subset of $X$ and $p \in \Omega$. Assume that $\mathcal{F}: \bar{\Omega} \rightarrow X$ is a compact operator. Then, either

(i) $\mathcal{F}$ has a fixed point in $\bar{\Omega}$, or

(ii) There exists $a u \in \partial \Omega$ and $\lambda \in(0,1)$ such that $u=\lambda \mathcal{F} u+(1-\lambda) p$.

Theorem 4.5 Let $\left(H_{1}\right)-\left(H_{3}\right)$ hold. Let $S$ be the positive constant from Lemma 4.3. Then problems (4.11) are solvable. If $u$ is a solution of (4.11) for some $n \in \mathbb{N}$, then $u$ satisfies (4.18) and (4.19) with the positive constant $M$ given in (4.15).

Proof. Let

$$
\Omega:=\left\{x \in C^{1}[0, T]:\|x\|_{\infty}<S T,\left\|x^{\prime}\right\|_{\infty}<S\right\} .
$$

Then $\Omega$ is an open bounded subset of the Banach space $C^{1}[0, T]$. Choose $n \in \mathbb{N}$ and consider an operator $\mathcal{K}:[0,1] \times \bar{\Omega} \rightarrow C^{1}[0, T]$,

$$
\mathcal{K}(\lambda, x)=\lambda \mathcal{F} x+(1-\lambda) p,
$$

where

$$
\begin{aligned}
&(\mathcal{F} x)(t)= t \frac{c T^{a+1}}{|a+1|}\left(T^{-a-1}-t^{-a-1}\right) \\
& \quad+t \int_{t}^{T} s^{-a-2}\left(\int_{s}^{T} \xi^{a+1} f_{n}\left(\xi, x(\xi), x^{\prime}(\xi)\right) \mathrm{d} \xi\right) \mathrm{d} s \\
& p=t \Delta \int_{t}^{T} s^{-a-2}\left(\int_{s}^{T} \xi^{a+1} \mathrm{~d} \xi\right) \mathrm{d} s .
\end{aligned}
$$


By Lemma 4.2, any fixed point of the operator $\mathcal{K}(1, \cdot)=\mathcal{F}$ is a solution of problem (4.11). Hence, to show the result we need to prove that $\mathcal{K}(1, \cdot)$ has a fixed point. Applying Lemma 4.4 for $X=C^{1}[0, T]$, we have to show that

(i) $\mathcal{K}(1, \cdot): \bar{\Omega} \rightarrow C^{1}[0, T]$ is a compact operator, and

(ii) $\mathcal{K}(\lambda, x) \neq x$ for each $\lambda \in(0,1)$ and $x \in \partial \Omega$.

We begin by proving the continuity of $\mathcal{K}(1, \cdot)$. To this end let $\left\{x_{m}\right\} \subset \bar{\Omega}$ be a convergent sequence and let $\lim _{m \rightarrow \infty} x_{m}=x$. Let

$$
r_{m}(t):=f_{n}\left(t, x_{m}(t), x_{m}^{\prime}(t)\right)-f_{n}\left(t, x(t), x^{\prime}(t)\right) \text { for a.e. } t \in[0, T] .
$$

Then (2.1) and (2.2) yield

$$
\begin{aligned}
\left|\mathcal{K}\left(1, x_{m}\right)(t)-\mathcal{K}(1, x)(t)\right| & =\left|t \int_{t}^{T} s^{-a-2}\left(\int_{s}^{T} \xi^{a+1} r_{m}(\xi) \mathrm{d} \xi\right) \mathrm{d} s\right| \leq \frac{T\left\|r_{m}\right\|_{1}}{|a+1|}, \\
\left|\mathcal{K}\left(1, x_{m}\right)^{\prime}(t)-\mathcal{K}(1, x)^{\prime}(t)\right|=\mid \int_{t}^{T} s^{-a-2}\left(\int_{s}^{T} \xi^{a+1} r_{m}(\xi) \mathrm{d} \xi\right) \mathrm{d} s & \quad-t^{-a-1} \int_{t}^{T} s^{a+1} r_{m}(s) \mathrm{d} s \mid \\
\leq & \left(\frac{1}{|a+1|}+1\right)\left\|r_{m}\right\|_{1},
\end{aligned}
$$

for $t \in[0, T]$ and $m \in \mathbb{N}$. Here, $\mathcal{K}(1, x)^{\prime}=\frac{\mathrm{d}}{\mathrm{d} t} \mathcal{K}(1, x)$. In particular,

$$
\begin{aligned}
\left\|\mathcal{K}\left(1, x_{m}\right)-\mathcal{K}(1, x)\right\|_{\infty} & \leq \frac{T\left\|r_{m}\right\|_{1}}{|a+1|} \\
\left\|\mathcal{K}\left(1, x_{m}\right)^{\prime}-\mathcal{K}(1, x)^{\prime}\right\|_{\infty} & \leq\left(\frac{1}{|a+1|}+1\right)\left\|r_{m}\right\|_{1},
\end{aligned}
$$

for $m \in \mathbb{N}$. If we show that $\lim _{m \rightarrow \infty}\left\|r_{m}\right\|_{1}=0$, then the above inequalities guarantee that $\mathcal{K}(1, \cdot)$ is a continuous operator. From

$$
\lim _{m \rightarrow \infty} f_{n}\left(t, x_{m}(t), x_{m}^{\prime}(t)\right)=f_{n}\left(t, x(t), x^{\prime}(t)\right) \text { for a.e. } t \in[0, T],
$$

and from the fact that $f_{n} \in \operatorname{Car}\left([0, T] \times \mathbb{R}^{2}\right)$ and $\left\{x_{m}\right\}$ is bounded in $C^{1}[0, T]$, it follows that

$$
\mid f_{n}\left(t, x_{m}(t), x_{m}^{\prime}(t) \mid \leq \rho(t) \text { for a.e. } t \in[0, T] \text { and all } m \in \mathbb{N},\right.
$$

where $\rho \in L^{1}[0, T]$. Finally, $\lim _{m \rightarrow \infty}\left\|r_{m}\right\|_{1}=0$ follows by the Lebesgue dominated convergence theorem.

Now, we show that the set $\mathcal{K}(1, \bar{\Omega})$ is relatively compact in $C^{1}[0, T]$. From $f_{n} \in \operatorname{Car}\left([0, T] \times \mathbb{R}^{2}\right)$ we conclude that

$$
\Delta \leq f_{n}\left(t, x(t), x^{\prime}(t)\right) \leq \mu(t) \text { for a.e. } t \in[0, T] \text { and all } x \in \bar{\Omega}
$$


where $\mu \in L^{1}[0, T]$. Then, by (2.1), (2.2), (4.25) and (4.26),

$$
\begin{aligned}
& 0 \leq \mathcal{K}(1, x)(t) \leq \frac{c T}{|a+1|}+t \int_{t}^{T} s^{-a-2}\left(\int_{s}^{T} \xi^{a+1} \mu(\xi) \mathrm{d} \xi\right) \mathrm{d} s \leq \frac{T}{|a+1|}\left(c+\|\mu\|_{1}\right) \\
&\left|\mathcal{K}(1, x)^{\prime}(t)\right| \leq\left|\frac{c T^{a+1}}{|a+1|}\left(T^{-a-1}+a t^{-a-1}\right)\right| \\
& \quad \quad+\int_{t}^{T} s^{-a-2}\left(\int_{s}^{T} \xi^{a+1} \mu(\xi) \mathrm{d} \xi\right) \mathrm{d} s+t^{-a-1} \int_{t}^{T} s^{a+1} \mu(s) \mathrm{d} s \\
& \leq\left(\frac{1}{|a+1|}+1\right)\left(c+\|\mu\|_{1}\right)
\end{aligned}
$$

for $t \in[0, T]$ and $x \in \bar{\Omega}$. Therefore, the set $\mathcal{K}(1, \bar{\Omega})$ is bounded in $C^{1}[0, T]$.

We now show that the set $\left\{\mathcal{K}(1, x)^{\prime}: x \in \bar{\Omega}\right\}$ is equicontinuous on $[0, T]$. For a.e. $t \in[0, T]$ and all $x \in \bar{\Omega}$ we have, by $(4.26)$,

$$
\begin{aligned}
\left|\mathcal{K}(1, x)^{\prime \prime}(t)\right|= & \mid a c T^{a+1} t^{-a-2}+a t^{-a-2} \int_{t}^{T} s^{a+1} f_{n}\left(s, x(s), x^{\prime}(s)\right) \mathrm{d} s \\
& \quad+f_{n}\left(t, x(t), x^{\prime}(t)\right) \mid \\
\leq & |a| c T^{a+1} t^{-a-2}+|a| t^{-a-2} \int_{t}^{T} s^{a+1} \mu(s) \mathrm{d} s+\mu(t) .
\end{aligned}
$$

Hence, by (2.1),

$$
\int_{0}^{T}\left|\mathcal{K}(1, x)^{\prime \prime}(t)\right| \mathrm{d} t \leq\left|\frac{a}{a+1}\right| c+\left|\frac{a}{a+1}\right|\|\mu\|_{1}+\|\mu\|_{1}
$$

for all $x \in \bar{\Omega}$, which guarantees the equicontinuity of the set $\left\{\mathcal{K}(1, x)^{\prime}: x \in \bar{\Omega}\right\}$ on $[0, T]$. Therefore, the set $\mathcal{K}(1, \bar{\Omega})$ is relatively compact in $C^{1}[0, T]$ by the Arzelà-Ascoli theorem and consequently, $\mathcal{K}(1, \cdot)$ is a compact operator and (i) follows.

It remains to prove (ii), that is, $\mathcal{K}(\lambda, x) \neq x$ for each $\lambda \in(0,1)$ and $x \in \partial \Omega$. Let $u$ be a fixed point of $\mathcal{K}(\lambda, \cdot)$ for some $\lambda \in(0,1)$. Then equalities (4.20) and (4.22) hold for $t \in[0, T]$. We see that $u$ satisfies (4.16b) and, as in the proof of Lemma 4.1, we conclude that $u$ is a solution of equation (4.16a). Therefore, Lemma 4.3 guarantees that $\mathcal{K}(\lambda, x) \neq x$ for $\lambda \in(0,1)$ and $x \in \partial \Omega$. By Lemma 4.4, problem (4.11) has a solution $u \in \bar{\Omega}$. Lemma 4.3 guarantees that $u$ satisfies (4.18) and (4.19).

The following result provides an important property of solutions of problem (4.11) which will be used in the proof of Theorem 5.1 in Section 5.

Lemma 4.6 Let $\left(H_{1}\right)-\left(H_{3}\right)$ hold. Let $u_{n}$ be a solution of problem (4.11). Then, the sequence $\left\{u_{n}^{\prime}\right\}$ is equicontinuous on $[0, T]$. 
Proof. By Theorem 4.5,

$$
u_{n}(t) \geq M t(T-t)^{2} \text { for } t \in[0, T] \text { and } n \in \mathbb{N},
$$

and

$$
\left\|u_{n}\right\|_{\infty}<S T, \quad\left\|u_{n}^{\prime}\right\|_{\infty}<S \quad \text { for } n \in \mathbb{N},
$$

where $S$ is a positive constant and $M$ is given in (4.15). Since $u_{n}$ is a fixed point of $\mathcal{K}(1, \cdot)$, cf. (4.25), the equality

$$
u_{n}^{\prime \prime}(t)=a c T^{a+1} t^{-a-2}+a t^{-a-2} \int_{t}^{T} s^{a+1} f_{n}\left(s, u_{n}(s), u_{n}^{\prime}(s)\right) \mathrm{d} s+f_{n}\left(t, u_{n}(t), u_{n}^{\prime}(t)\right)
$$

holds for a.e. $t \in[0, T]$ and all $n \in \mathbb{N}$. Owing to (4.1), (4.2), (4.27) and (4.28) we have

$\Delta \leq f_{n}\left(t, u_{n}(t), u_{n}^{\prime}(t)\right) \leq h(t, 1+S T, S)+\rho(t)$ for a.e. $t \in[0, T]$ and all $n \in \mathbb{N}$, where $\rho(t)=g\left(M t(T-t)^{2}\right)$ for $t \in(0, T)$. Let us choose $\chi(t):=h(t, 1+S T, S)+$ $\varrho(t)$ for a.e. $t \in[0, T]$. By $\left(H_{3}\right)$ and Remark 1.1, $\chi$ is positive and $\chi \in L^{1}[0, T]$. Hence,

$$
\left|u_{n}^{\prime \prime}(t)\right| \leq|a| c T^{a+1} t^{-a-2}+|a| t^{-a-2} \int_{t}^{T} s^{a+1} \chi(s) \mathrm{d} s+\chi(t)
$$

for a.e. $t \in[0, T]$ and all $n \in \mathbb{N}$. Consequently, by (2.2), the inequality

$$
\int_{0}^{T}\left|u_{n}^{\prime \prime}(t)\right| \mathrm{d} s \leq\left|\frac{a}{a+1}\right| c+\left|\frac{a}{a+1}\right|\|\chi\|_{1}+\|\chi\|_{1}
$$

holds for all $n \in \mathbb{N}$, which means that that the sequence $\left\{u_{n}^{\prime}\right\}$ is equicontinuous on $[0, T]$.

\section{Analytical properties of solutions to problem $(1.1)$}

In this section, we denote by $\mathcal{L}$ the set of all positive solutions of the singular Dirichlet problem (1.1). For $c \geq 0$, we denote by $\mathcal{S}_{c}$ the set of all positive solutions of problem (1.1) satisfying condition (4.12). Our aim is to describe the structure of $\mathcal{L}$. In particular, we show that $\mathcal{L}$ is a one parameter set. 
Theorem 5.1 Let $\left(H_{1}\right)-\left(H_{3}\right)$ hold. Then, for each $c \geq 0$, the set $\mathcal{S}_{c}$ is nonempty and

$$
\mathcal{L}=\bigcup_{0 \leq c} \mathcal{S}_{c}
$$

Hence the cardinality of the set $\mathcal{L}$ is a continuum. Moreover,

$$
u(t) \geq M t(T-t)^{2}, \quad t \in[0, T],
$$

with $M$ given in (4.15), holds for each $u \in \mathcal{L}$.

Proof. Let us fix $c \geq 0$. Theorem 4.5 guarantees that the regular problem (4.11) has a solution $u_{n}$ satisfying inequalities (4.27) and (4.28), where $S$ is a positive constant and $M$ is given in (4.15). Furthermore, $\left\{u_{n}^{\prime}\right\}$ is equicontinuous on $[0, T]$ by Lemma 4.6. Consequently, by Arzelà-Ascoli theorem, there is a subsequence $\left\{u_{\ell_{n}}\right\}$ of $\left\{u_{n}\right\}$ converging in $C^{1}[0, T]$. Denote $\lim _{n \rightarrow \infty} u_{\ell_{n}}=: u$. Then $u$ satisfies the boundary conditions (4.11b) and taking the limit $n \rightarrow \infty$ in (4.27) and (4.28), with $u_{n}$ replaced by $u_{\ell_{n}}$, we obtain

$$
\|u\|_{\infty} \leq S T,\left\|u^{\prime}\right\|_{\infty} \leq S \text { and } u(t) \geq M t(T-t)^{2} \text { for } t \in[0, T] .
$$

Hence $u>0$ on $(0, T)$ and

$$
\lim _{n \rightarrow \infty} f_{\ell_{n}}\left(t, u_{\ell_{n}}(t), u_{\ell_{n}}^{\prime}(t)\right)=f\left(t, u(t), u^{\prime}(t)\right) \text { for a.e. } t \in[0, T] .
$$

By $\left(H_{3}\right)$ and Remark 1.1,

$$
\left|f_{\ell_{n}}\left(t, u_{\ell_{n}}(t), u_{\ell_{n}}^{\prime}(t)\right)\right| \leq h(t, 1+S T, S)+g\left(M t(T-t)^{2}\right) \in L^{1}[0, T] .
$$

Therefore, $f\left(t, u(t), u^{\prime}(t)\right) \in L^{1}[0, T]$ and

$$
\lim _{n \rightarrow \infty}\left\|f_{\ell_{n}}\left(t, u_{\ell_{n}}(t), u_{\ell_{n}}^{\prime}(t)\right)-f\left(t, u(t), u^{\prime}(t)\right)\right\|_{1}=0
$$

by the Lebesgue dominated convergence theorem. It follows from the inequality (2.2), that it holds

$$
\begin{aligned}
& \left|t \int_{t}^{T} s^{-a-2}\left(\int_{s}^{T} \xi^{a+1}\left[f_{\ell_{n}}\left(\xi, u_{\ell_{n}}(\xi), u_{\ell_{n}}^{\prime}(\xi)\right)-f\left(\xi, u(\xi), u^{\prime}(\xi)\right)\right] \mathrm{d} \xi\right) \mathrm{d} s\right| \\
& \quad \leq \frac{T}{|a+1|}\left\|f_{\ell_{n}}\left(t, u_{\ell_{n}}(t), u_{\ell_{n}}^{\prime}(t)\right)-f\left(t, u(t), u^{\prime}(t)\right)\right\|_{1} .
\end{aligned}
$$

Now, from (5.2) we conclude that

$$
\begin{array}{r}
\lim _{n \rightarrow \infty} \int_{t}^{T} s^{-a-2}\left(\int_{s}^{T} \xi^{a+1} f_{\ell_{n}}\left(\xi, u_{\ell_{n}}(\xi), u_{\ell_{n}}^{\prime}(\xi)\right) \mathrm{d} \xi\right) \mathrm{d} s \\
=\int_{t}^{T} s^{-a-2}\left(\int_{s}^{T} \xi^{a+1} f\left(\xi, u(\xi), u^{\prime}(\xi) \mathrm{d} \xi\right) \mathrm{d} s\right.
\end{array}
$$


is satisfied for $t \in[0, T]$. Letting $n \rightarrow \infty$ in

$$
u_{\ell_{n}}(t)=t \frac{c T^{a+1}}{|a+1|}\left(T^{-a-1}-t^{-a-1}\right)+t \int_{t}^{T} s^{-a-2}\left(\int_{s}^{T} \xi^{a+1} f_{\ell_{n}}\left(\xi, u_{\ell_{n}}(\xi), u_{\ell_{n}}^{\prime}(\xi)\right) \mathrm{d} \xi\right) \mathrm{d} s
$$

yields

$$
u(t)=t \frac{c T^{a+1}}{|a+1|}\left(T^{-a-1}-t^{-a-1}\right)+t \int_{t}^{T} s^{-a-2}\left(\int_{s}^{T} \xi^{a+1} f\left(\xi, u(\xi), u^{\prime}(\xi) \mathrm{d} \xi\right) \mathrm{d} s\right.
$$

for $t \in[0, T]$. A direct computation shows that $u$ is a solution of (1.1a). This means that $u$ is a positive solution of problem (1.1) and (4.12), that is $u \in \mathcal{S}_{c}$. Consequently $\mathcal{S}_{c} \neq \emptyset$. Since for each positive solution $u$ of problem (1.1) there exists $c=c(u) \geq 0$ such that $u^{\prime}(T)=-c$, we see that $\mathcal{L}=\bigcup_{0 \leq c} \mathcal{S}_{c}$ is the set of all positive solutions of problem (1.1).

For $K \geq 0$, let us denote

$$
\mathcal{L}_{K}:=\bigcup_{0 \leq c \leq K} \mathcal{S}_{c}
$$

Then we have the following theorem.

Theorem 5.2 Let $\left(H_{1}\right)-\left(H_{3}\right)$ hold. Then, for each $K \geq 0$, the set $\mathcal{L}_{K}$ is compact in $C^{1}[0, T]$.

Proof. Let us choose $K \geq 0$. Then inequality (5.1) with $M$ given in (4.15) holds for each $u \in \mathcal{L}_{K}$. Consider an arbitrary $u \in \mathcal{L}_{K}$. Then (5.3) is satisfied for some $c=c(u) \in[0, K]$ and $t \in[0, T]$. Therefore,

$$
\begin{aligned}
& u^{\prime}(t)=\frac{c T^{a+1}}{|a+1|}\left(T^{-a-1}+a t^{-a-1}\right) \\
& \quad+\int_{t}^{T} s^{-a-2}\left(\int_{s}^{T} \xi^{a+1} f\left(\xi, u(\xi), u^{\prime}(\xi)\right) \mathrm{d} \xi\right) \mathrm{d} s \\
& \quad-t^{-a-1} \int_{t}^{T} s^{a+1} f\left(s, u(s), u^{\prime}(s)\right) \mathrm{d} s, \quad t \in[0, T] .
\end{aligned}
$$

It follows from (2.1) and (2.2),

$$
\left|u^{\prime}(t)\right| \leq K\left(\frac{1}{|a+1|}+1\right)+\left(\frac{1}{|a+1|}+1\right) \int_{t}^{T} f\left(s, u(s), u^{\prime}(s)\right) \mathrm{d} s, \quad t \in[0, T] .
$$

Hence, by $\left(H_{3}\right)$ and $\|u\|_{\infty} \leq T\left\|u^{\prime}\right\|_{\infty}$, since $u(t)=\int_{0}^{t} u^{\prime}(s) \mathrm{d} s$, we have

$$
\begin{aligned}
\left|u^{\prime}(t)\right| \leq & \left(\frac{1}{|a+1|}+1\right)\left(K+\int_{t}^{T}\left(h\left(s, u(s),\left|u^{\prime}(s)\right|\right)+g(u(s))\right) \mathrm{d} s\right) \\
& \leq\left(\frac{1}{|a+1|}+1\right)\left(K+\int_{0}^{T} h\left(s, T\left\|u^{\prime}\right\|_{\infty},\left\|u^{\prime}\right\|_{\infty}\right) \mathrm{d} s+W\right), t \in[0, T] .
\end{aligned}
$$


Note that by Remark 1.1,

$$
\int_{0}^{T} g(u(t)) \mathrm{d} t \leq \int_{0}^{T} g\left(M t(T-t)^{2}\right) d t=: W<\infty .
$$

In particular,

$$
1 \leq \frac{1}{\left\|u^{\prime}\right\|_{\infty}}\left(\frac{1}{|a+1|}+1\right)\left(K+\int_{0}^{T} h\left(s, T\left\|u^{\prime}\right\|_{\infty},\left\|u^{\prime}\right\|_{\infty}\right) \mathrm{d} s+W\right) .
$$

Due to $\left(H_{3}\right)$,

$$
\lim _{w \rightarrow \infty} \frac{1}{w} \int_{0}^{T} h(\xi, T w, w) d \xi=0
$$

and so

$$
\lim _{w \rightarrow \infty} \frac{1}{w}\left(\frac{1}{|a+1|}+1\right)\left(K+\int_{0}^{T} h(s, T w, w) \mathrm{d} s+W\right)=0
$$

which implies that there exists $\varrho^{*}>0$ such that

$$
\frac{1}{w}\left(\frac{1}{|a+1|}+1\right)\left(K+\int_{0}^{T} h(s, T w, w) \mathrm{d} s+W\right)<1
$$

for each $w \geq \varrho^{*}$. This together with (5.5) results in

$$
\left\|u^{\prime}\right\|_{\infty}<\varrho^{*}, \quad\|u\|_{\infty}<\varrho^{*} T
$$

for each $u \in \mathcal{L}_{K}$ and therefore, $\mathcal{L}_{K}$ is bounded in $C^{1}[0, T]$.

We now verify that the set $\left\{u^{\prime}: u \in \mathcal{L}_{K}\right\}$ is equicontinuous on $[0, T]$. For any $u \in \mathcal{L}_{K}$ we have

$$
\begin{aligned}
u^{\prime \prime}(t)= & a c T^{a+1} t^{-a-2}+a t^{-a-2} \int_{t}^{T} s^{a+1} f\left(s, u(s), u^{\prime}(s) \mathrm{d} s\right. \\
& +f\left(t, u(t), u^{\prime}(t)\right) \text { for a.e. } t \in[0, T] \text { and } c=c(u) \in[0, K] .
\end{aligned}
$$

Therefore,

$$
\left|u^{\prime \prime}(t)\right| \leq|a| K T^{a+1} t^{-a-2}+|a| t^{-a-2} \int_{t}^{T} s^{a+1} m(s) \mathrm{d} s+m(t)
$$

for a.e. $t \in[0, T]$, where $m(t)=h\left(t, T \varrho^{*}, \varrho^{*}\right)+g\left(M t(T-t)^{2}\right)$. Since, cf. (5.4) and $\left(H_{3}\right)$,

$$
\int_{0}^{T} m(t) \mathrm{d} t \leq \int_{0}^{T} h\left(t, T \varrho^{*}, \varrho^{*}\right) \mathrm{d} t+W<\infty
$$


we see that $m \in L^{1}[0, T]$. Therefore, by $(2.2)$,

$$
t^{-a-2}\left(\int_{t}^{T} s^{a+1} m(s) \mathrm{d} s\right) \in L^{1}[0, T] .
$$

Consequently, there exists a majorant function $p^{*} \in L^{1}[0, T]$ satisfying

$$
\left|u^{\prime \prime}(t)\right| \leq p^{*}(t) \quad \text { for a.e. } t \in[0, T] \text { and all } u \in \mathcal{L}_{K} .
$$

As a result the set $\left\{u^{\prime}: u \in \mathcal{L}_{K}\right\}$ is equicontinuous on $[0, T]$.

In order to complete the proof, we need to show that the set $\mathcal{L}_{K}$ is closed in $C^{1}[0, T]$. To this end, we consider a sequence $\left\{u_{n}\right\} \subset \mathcal{L}_{K}$ converging in $C^{1}[0, T]$ to a function $u \in C^{1}[0, T]$. Therefore, there exists a sequence $\left\{c_{n}\right\} \subset[0, K]$ such that, due to (5.3),

$$
\begin{aligned}
u_{n}(t)= & t \frac{c_{n} T^{a+1}}{|a+1|}\left(T^{-a-1}-t^{-a-1}\right) \\
& +t \int_{t}^{T} s^{-a-2}\left(\int_{s}^{T} \xi^{a+1} f\left(\xi, u_{n}(\xi), u_{n}^{\prime}(\xi) \mathrm{d} \xi\right) \mathrm{d} s \text { for } t \in[0, T] \text { and } n \in \mathbb{N} .\right.
\end{aligned}
$$

Since $u_{n}^{\prime}(T)=-c_{n}$, we see that $\left\{c_{n}\right\}$ is convergent. Let us define $\lim _{n \rightarrow \infty} c_{n}=$ : $c \in[0, K]$ and let $n \rightarrow \infty$ in the above equality for $u_{n}(t)$. Then, by the Lebesgue dominated convergence theorem, arguing as in the proof of Theorem 5.1, we obtain

$$
\begin{aligned}
u(t)= & t \frac{c T^{a+1}}{|a+1|}\left(T^{-a-1}-t^{-a-1}\right) \\
& +t \int_{t}^{T} s^{-a-2}\left(\int_{s}^{T} \xi^{a+1} f\left(\xi, u(\xi), u^{\prime}(\xi) \mathrm{d} \xi\right) \mathrm{d} s \quad \text { for } t \in[0, T] .\right.
\end{aligned}
$$

Therefore $u$ satisfies (1.1) and (4.12) and hence $u \in \mathcal{S}_{c} \subset \mathcal{L}_{K}$.

Remark 5.3 It follows from the proof of Theorem 5.2 that for each fixed $c \geq 0$ the set $\mathcal{S}_{c}$ is compact in $C^{1}[0, T]$.

Remark 5.4 Consider a sequence $\left\{u_{n}\right\} \subset \mathcal{L}$. Since $\mathcal{L}=\cup_{0 \leq c} \mathcal{S}_{c}$, we see that $u_{n} \in \mathcal{S}_{c_{n}}$ for some $c_{n} \geq 0$ and $u_{n}^{\prime}(T)=-c_{n}, n \in \mathbb{N}$. We now can show that

$$
\lim _{n \rightarrow \infty}\left\|u_{n}\right\|_{C^{1}}=\infty \quad \Longleftrightarrow \quad \lim _{n \rightarrow \infty} c_{n}=\infty
$$

holds. First, assume that

$$
\lim _{n \rightarrow \infty}\left\|u_{n}\right\|_{C^{1}}=\infty
$$


and further, assume in a contrary, that there exist $K>0$ and a subsequence $\left\{c_{m_{n}}\right\} \subset\left\{c_{n}\right\}$ such that

$$
c_{m_{n}} \leq K \quad \text { for } n \in \mathbb{N} \text {. }
$$

Since $\mathcal{L}_{K}=\cup_{0 \leq c \leq K} \mathcal{S}_{c}$, we have $\left\{u_{m_{n}}\right\} \subset \mathcal{L}_{K}$. By Theorem 5.2, $\mathcal{L}_{K}$ is compact in $C^{1}[0, T]$ which implies that the sequence $\left\{u_{m_{n}}\right\}$ is bounded in $C^{1}[0, T]$, which contradicts $(5.8)$.

If we assume that $\lim _{n \rightarrow \infty} c_{n}=\infty$, then (5.8) immediately follows, because $\left\|u_{n}\right\|_{C^{1}} \geq\left\|u_{n}^{\prime}\right\|_{\infty} \geq c_{n}, n \in \mathbb{N}$.

Remark 5.5 It follows from (5.7) that the set $\mathcal{L}$ is unbounded in $C^{1}[0, T]$. In particular, if $u \in \mathcal{L}$, then $u \in \mathcal{S}_{c}$ for some $c \geq 0$ and, keeping in mind that $f$ is positive, we get by (5.6),

$$
u\left(\frac{T}{2}\right) \geq \frac{T c}{2|a+1|}\left(1-\frac{1}{2^{-a-1}}\right) .
$$

That is $\|u\|_{\infty} \geq c L$, where

$$
L=\frac{T}{2|a+1|}\left(1-\frac{1}{2^{-a-1}}\right)>0 .
$$

Hence,

$$
\sup \left\{\|u\|_{\infty}: u \in \mathcal{L}\right\} \geq \sup \{c L: c \in[0, \infty)\}=\infty,
$$

and Theorem 5.2 implies the following results concerning minimal values of functionals defined on the set $\mathcal{L}$ of all positive solutions to problem (1.1).

Let $\mathcal{M}$ be the set of continuous functionals $\Phi: C^{1}[0, T] \rightarrow[0, \infty)$, which are coercive on $\mathcal{L}$, that is,

$$
\lim _{x \in \mathcal{L},\|x\|_{C^{1} \rightarrow \infty}} \Phi(x)=\infty .
$$

Theorem 5.6 Let $\left(H_{1}\right)-\left(H_{3}\right)$ hold and let $\Phi \in \mathcal{M}$. Then there exists a positive solution $u_{*}$ of problem (1.1) such that

$$
\min \{\Phi(x): x \in \mathcal{L}\}=\Phi\left(u_{*}\right) .
$$

Proof. Choose $u_{0} \in \mathcal{S}_{0}$ and $A:=\Phi\left(u_{0}\right)$. By (5.10), there exists $B>0$ such that

$$
x \in \mathcal{L},\|x\|_{C^{1}}>B \quad \Rightarrow \quad \Phi(x)>A .
$$


Let $K:=B /(L+1)$, with $L>0$ from (5.9). According to Theorem $5.2, \mathcal{L}_{K}$ is compact in $C^{1}[0, T]$ and consequently, the continuity of $\Phi$ implies the existence of $u_{*} \in \mathcal{L}_{K}$ such that

$$
\min \left\{\Phi(x): x \in \mathcal{L}_{K}\right\}=\Phi\left(u_{*}\right) \leq A .
$$

Now, assume that $c>K$. Then, for each $x \in \mathcal{S}_{c}$, we have $\left\|x^{\prime}\right\|_{\infty} \geq\left|x^{\prime}(T)\right|=c$ and, by Remark 5.5, $\|x\|_{\infty} \geq c L$. Therefore,

$$
\|x\|_{C^{1}} \geq c(L+1)>K(L+1)=B .
$$

This together with (5.12) yields $\Phi(x)>A$. Consequently, $\Phi(x)>A$ for each $x \in \mathcal{L} \backslash \mathcal{L}_{K}$ and (5.11) follows from (5.13).

We now present applications of Theorem 5.6. Assume $\left(H_{1}\right)-\left(H_{3}\right)$ to hold. Choose $\alpha \in(0, \infty)$ and consider functionals $\Phi_{1}, \Phi_{2}: C^{1}[0, T] \rightarrow[0, \infty)$ given by

$$
\Phi_{1}(x)=\int_{0}^{T}|x(t)|^{\alpha} \mathrm{d} t, \quad \Phi_{2}(x)=\int_{0}^{T} \sqrt{1+x^{\prime 2}(t)} \mathrm{d} t .
$$

Then, $\Phi_{1}, \Phi_{2}$ are continuous on $C^{1}[0, T]$.

Let us now show that $\Phi_{1}$ and $\Phi_{2}$ satisfy (5.10), where $\mathcal{L}$ is the set of all positive solutions of problem (1.1). Choose an arbitrary sequence $\left\{u_{n}\right\} \subset \mathcal{L}$ such that $\lim _{n \rightarrow \infty}\left\|u_{n}\right\|_{C^{1}}=\infty$. For $c_{n}=-u_{n}^{\prime}(T), n \in \mathbb{N}$, we obtain using (5.7), $\lim _{n \rightarrow \infty} c_{n}=\infty$. Since $u_{n}(t)=\int_{0}^{t} u_{n}^{\prime}(s) \mathrm{d} s$, it follows from Remark 5.5 that

$$
c_{n} L \leq\left\|u_{n}\right\|_{\infty} \leq \int_{0}^{T}\left|u_{n}^{\prime}(t)\right| \mathrm{d} t \leq \Phi_{2}\left(u_{n}\right), \quad n \in \mathbb{N} .
$$

Consequently, $\Phi_{2}$ is coercive on $\mathcal{L}$.

Furthermore, from (5.6) and the positivity of $f$, we have

$$
u_{n}(t) \geq c_{n} \varphi(t), \quad t \in[0, T]
$$

where

$$
\varphi(t):=t \frac{T^{a+1}}{|a+1|}\left(T^{-a-1}-t^{-a-1}\right)>0, \quad t \in(0, T) .
$$

According to (5.14),

$$
\Phi_{1}\left(u_{n}\right)=\int_{0}^{T}\left|u_{n}(t)\right|^{\alpha} \mathrm{d} t \geq c_{n}^{\alpha} \int_{0}^{T} \varphi^{\alpha}(t) \mathrm{d} t=c_{n}^{\alpha} M_{0}, n \in \mathbb{N},
$$

where $M_{0}:=\int_{0}^{T} \varphi^{\alpha}(t) \mathrm{d} t>0$. Hence, we have shown that $\lim _{n \rightarrow \infty} \Phi_{1}\left(u_{n}\right)=\infty$ and therefore, $\Phi_{1}$ is coercive on $\mathcal{L}$.

Consequently, Theorem 5.6 is applicable to both, $\Phi_{1}$ and $\Phi_{2}$. We can easily see a geometrical meaning of this result. For example, dealing with $\Phi_{2}$, we get that among all positive solutions of (1.1) there exists a solution having a graph with the shortest lenght. Note, that values of $\Phi_{1}$ with $\alpha=1$ are discussed in Example 3. 


\section{$6 \quad$ Numerical simulations}

For the numerical simulation, we use an alternative formulation of problem (1.1),

$$
\begin{aligned}
& u^{\prime \prime}(t)+\frac{a}{t} u^{\prime}(t)-\frac{a}{t^{2}} u(t)=f\left(t, u(t), u^{\prime}(t)\right), \quad t \in[0,1] \\
& u(1)=0, \quad u^{\prime}(1)=-c
\end{aligned}
$$

where $c \geq 0$. According to [19], the above boundary value problem (6.1) is well-posed and therefore it is suitable for the numerical treatment. For all examples, the calculations have been carried for the values of $a=-2$ and $c=0,0.1,0.2,0.3,0.5,1,2,5,10,100$.

\subsection{Matlab Code bvpsuite}

To illustrate the analytical results discussed in the previous section, we solved numerically Examples (6.2), (6.4) and (6.5) using a MATLAB ${ }^{\mathrm{TM}}$ software package bvpsuite designed to solve boundary value problems in ordinary differential equations and differential algebraic equations. The solver routine is based on a class of collocation methods whose orders may vary from 2 to 8 . Collocation has been investigated in context of singular differential equations of first and second order in [11] and [20], respectively. This method could be shown to be robust with respect to singularities in time and for retains its high convergence order in case that the analytical solution is appropriately smooth. The code also provides an asymptotically correct estimate for the global error of the numerical approximation. To enhance the efficiency of the method, a mesh adaptation strategy is implemented, which attempts to choose grids related to the solution behavior, in such a way that the tolerance is satisfied with the least possible effort. Error estimate procedure and the mesh adaptation work dependably provided that the solution of the problem and its global error are appropriately smooth ${ }^{2}$. The code and the manual can be downloaded from http://www. math.tuwien.ac.at/ ewa. For further information see [13]. This software is useful for the approximation of numerous singular boundary value problems important for applications, see e.g. [4], [9], [12], [17].

\subsection{Example 1}

We first investigate the following problem:

$$
\begin{aligned}
& u^{\prime \prime}(t)+\frac{a}{t} u^{\prime}(t)-\frac{a}{t^{2}} u(t)=u(t)^{\frac{2}{3}}+t+1, \quad t \in[0,1], \\
& u(1)=0, \quad u^{\prime}(1)=-c,
\end{aligned}
$$

\footnotetext{
${ }^{2}$ The required smoothness of higher derivatives is related to the order of the used collocation method.
} 
where $a=-2$. In Figures 1 and 2, solutions to problem (6.2) for different values of $c$ are shown.

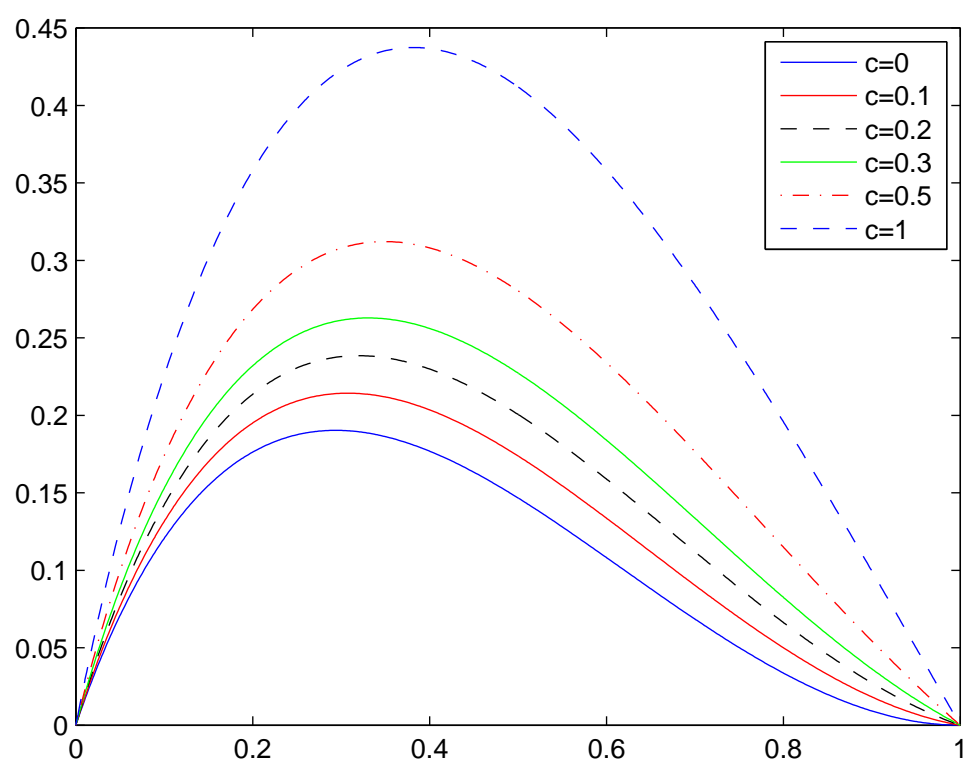

Figure 1: Problem (6.2): Parameter $a=-2$.

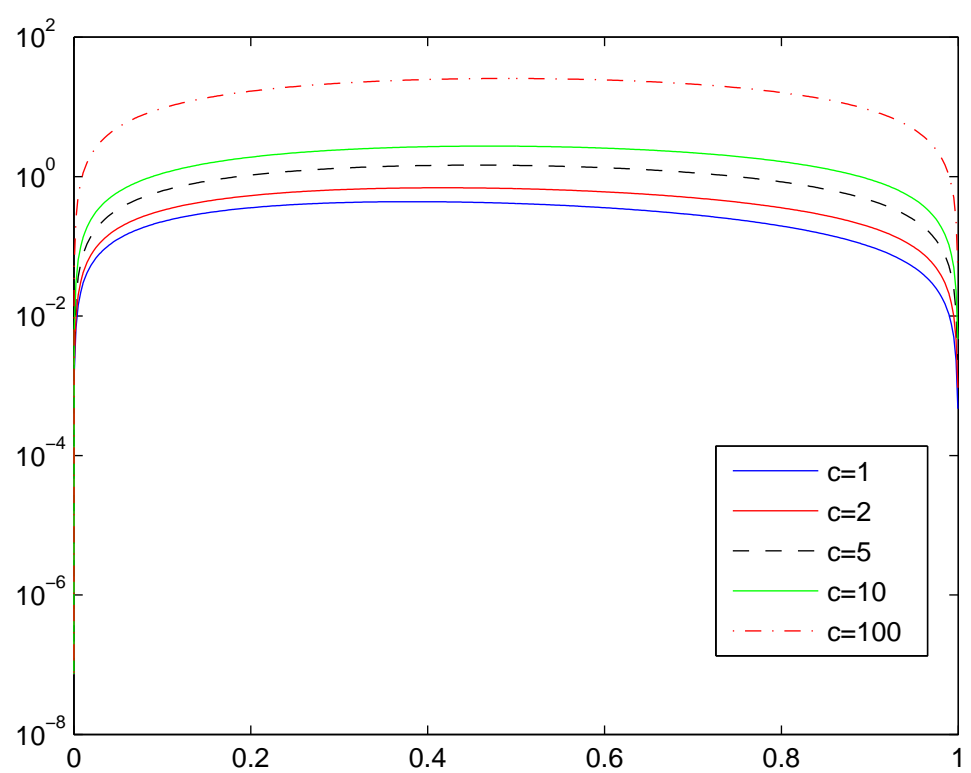

Figure 2: Problem (6.2): Parameter $a=-2$. 
For a given $c \geq 0$ denote by $u_{c}$ a solution of problem (6.2). We can see in Figures 1 and 2 that graphs of solutions $u_{c}$ are ordered, that is

$$
c_{1}<c_{2} \quad \Longrightarrow \quad u_{c_{1}}(t)<u_{c_{2}}(t), \quad t \in(0,1) .
$$

This corresponds to the theory in Section 4 of [16], where the special case of problem (1.1) with $f \in \operatorname{Car}([0, T] \times[0, \infty))$ and $f(t, u)$ increasing in $u$, has been investigated.

\subsection{Example 2}

Here, we study the influence of $u^{\prime}$ in $f\left(t, u, u^{\prime}\right)=u^{\frac{2}{3}}+u^{\prime \frac{2}{3}}+t+1$. The boundary value problem has now the form

$$
\begin{aligned}
& u^{\prime \prime}(t)+\frac{a}{t} u^{\prime}(t)-\frac{a}{t^{2}} u(t)=u(t)^{\frac{2}{3}}+u^{\prime}(t)^{\frac{2}{3}}+t+1, \quad t \in[0,1], \\
& u(1)=0, \quad u^{\prime}(1)=-c
\end{aligned}
$$

where $a=-2$. Note that since $u^{\prime}(t)$ may become negative, we replace $u^{\prime}(t)^{\frac{2}{3}}$ by $u^{\prime}(t)\left|u^{\prime}(t)\right|^{-\frac{1}{3}}$ in numerical simulations.

The solutions of the boundary value problem (6.4) for different values of $c$, can be found in Figures 3 and 4.

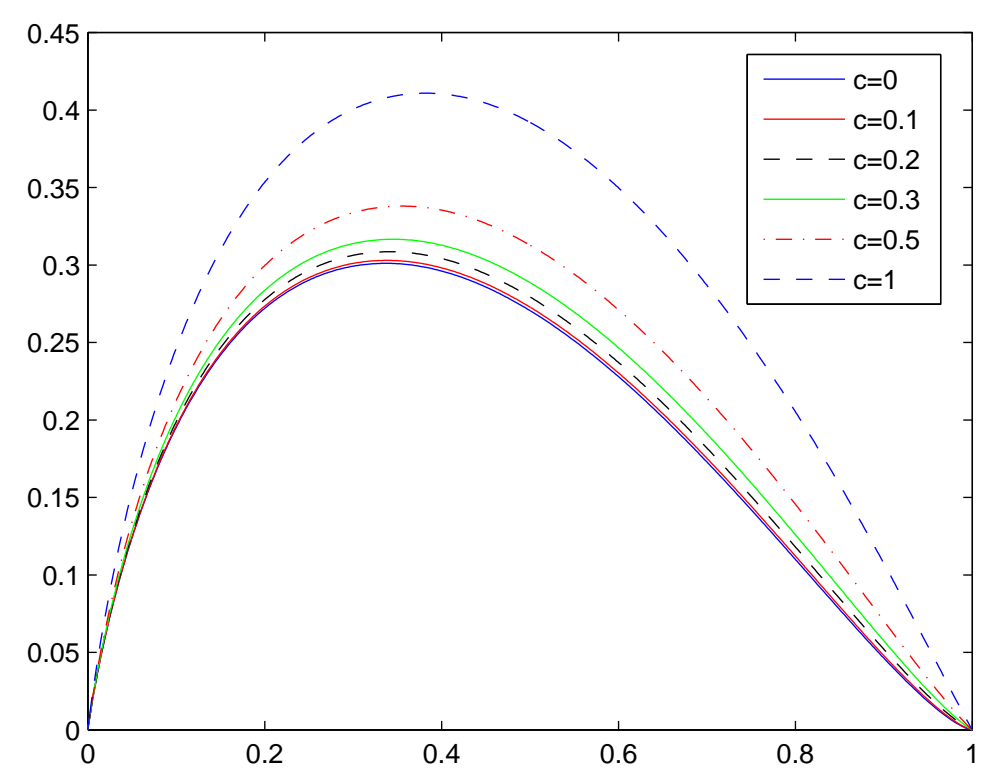

Figure 3: Problem (6.4): Parameter $a=-2$. 


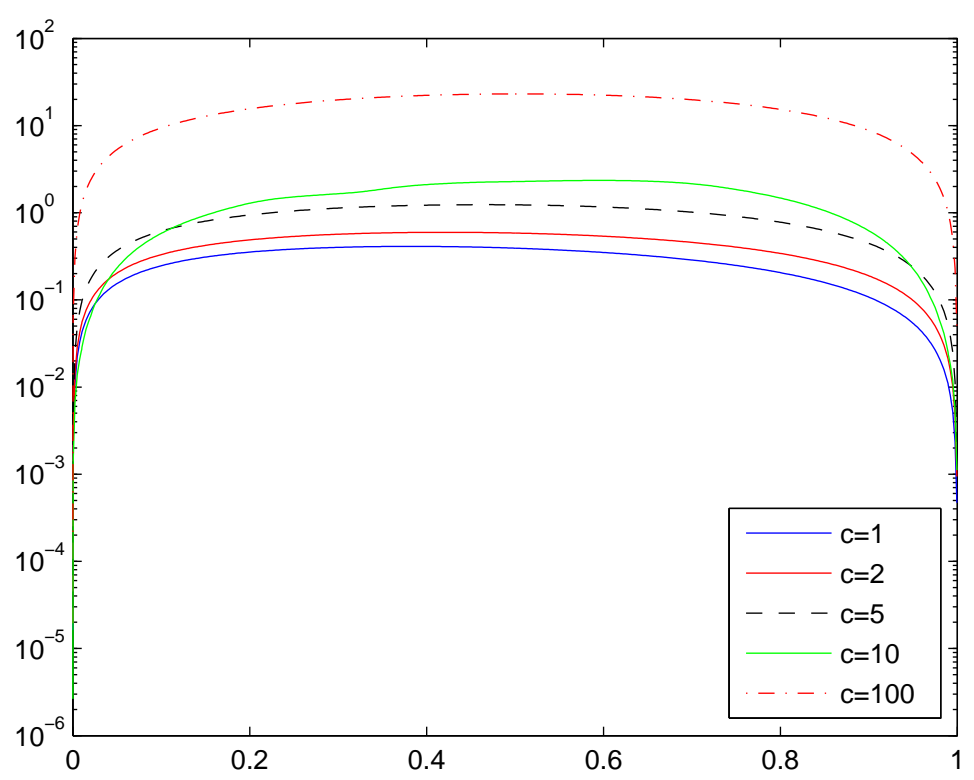

Figure 4: Problem (6.4): Parameter $a=-2$.

Figure 3 shows ordered graphs of solutions $u_{c}$ of problem (6.4) with $c$ changing from 0 to 1 but Figure 4 demonstrates that, for $c$ having values from 1 to 100, the graphs of solutions $u_{c}$ do not keep order (6.3).

The solutions of problem (6.4) for $a=-3$ and $a=-10$ show similar behavior as for $a=-2$ and hence, they are not displayed here. All results for the above class have been obtained using the same starting guess: the numerical solution for $c=0$ obtained with the piecewise hat function as an initial profile, see Figure 5 . 


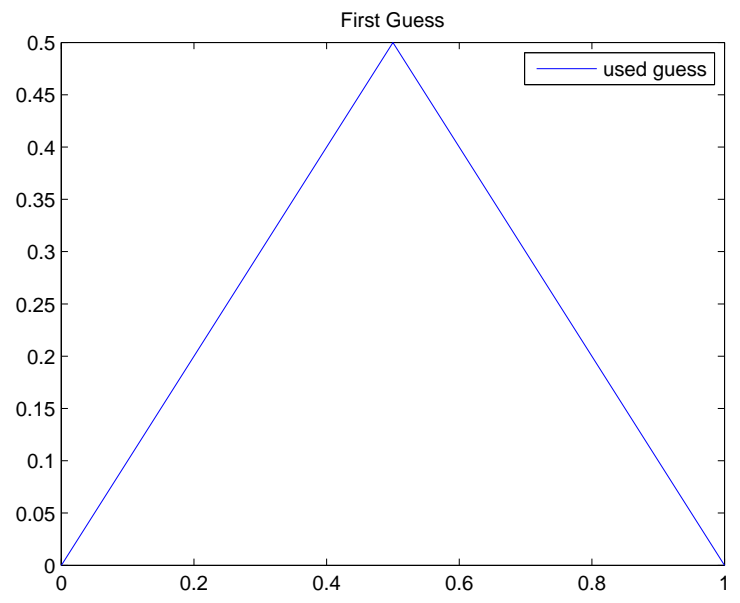

Figure 5: Starting guess for $c \neq 0$ has been obtained by solving the problem for $c=0$ and the initial profile shown above.

\subsection{Example 3}

In order to discuss the influence of a possible space singularity in $f$, we put $f(t, u)=u^{-\frac{1}{3}}+t+1$ and look at the following problem:

$$
\begin{aligned}
& u^{\prime \prime}(t)+\frac{a}{t} u^{\prime}(t)-\frac{a}{t^{2}} u(t)=u(t)^{-\frac{1}{3}}+t+1, \quad t \in[0,1] \\
& u(1)=0, \quad u^{\prime}(1)=-c
\end{aligned}
$$

where $a=-2$. The solutions to the above boundary value problem can be found in Figures 6 and 7. 


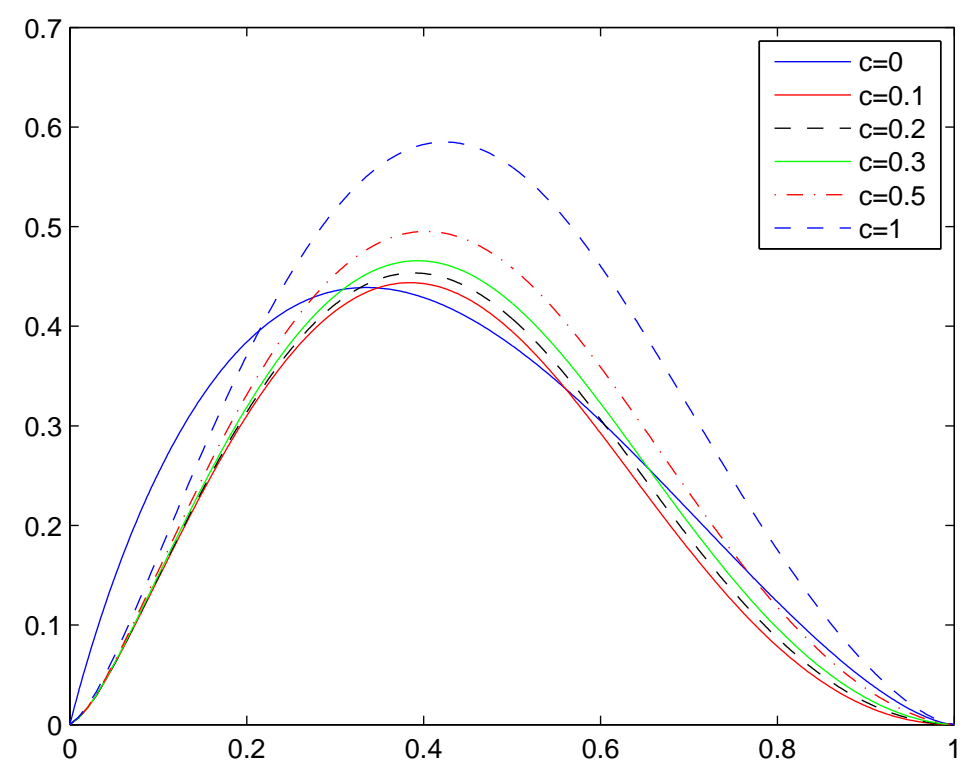

Figure 6: Problem (6.5): Parameter $a=-2$.

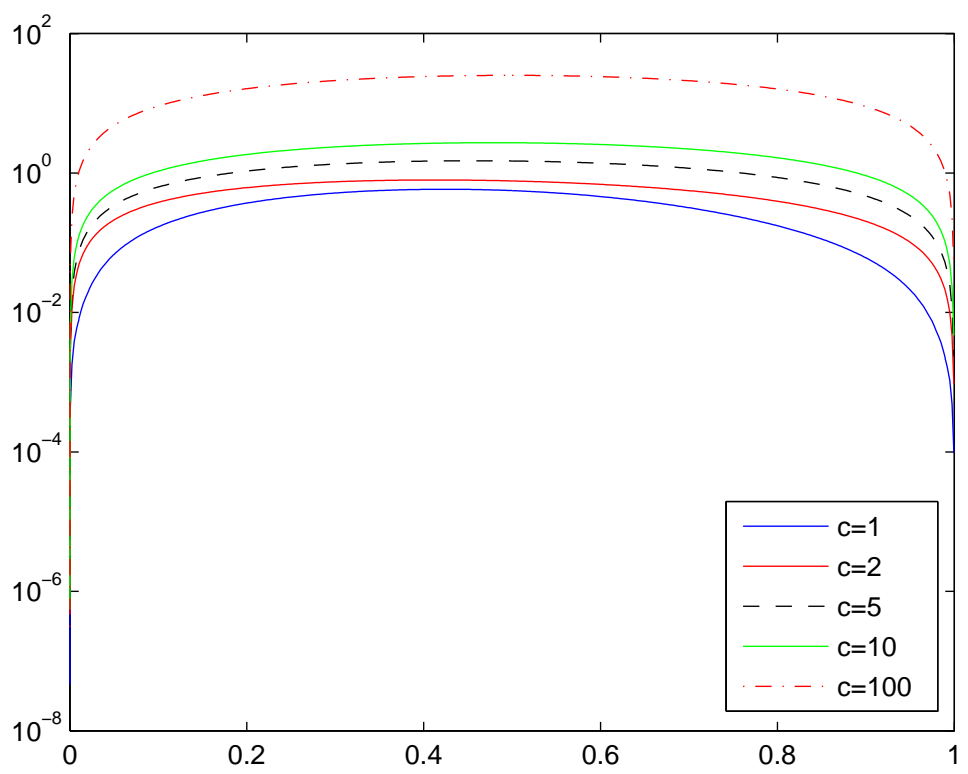

Figure 7: Problem (6.5): Parameter $a=-2$.

For the values of $c \geq 1$ the starting guess mentioned above has been used. For larger values of $c$ providing alternative starting guesses was necessary. For 
example, for $c=5$ the earlier computed solution for $c=2$ has been used.

Figure 6 illustrates that solutions $u_{c}$ of problem (6.5) with $c$ changing from 0 to 1 do not fulfil the order given by (6.3). This together with Figure 4 leads to the hypothesis that the order (6.3) cannot be proved for solutions of (1.1), where $f(t, x, y)$ depends on $y$ or $f$ has a singularity at $x=0$.

Now, consider the functional $\Phi_{1}$ of (5.14) with $\alpha=1$ and $T=1$, that is $\Phi_{1}(x)=\int_{0}^{1}|x(t)| \mathrm{d} t$ for $x \in C^{1}[0,1]$. Let $\mathcal{L}$ be the set of all positive solutions of problem (6.5). Using Theorem 5.6, we have proved that there exists a positive solution of problem (6.5) giving a minimal value of $\Phi_{1}$ on $\mathcal{L}$. To illustrate this result, we have approximated the values of the integrals

$$
\Phi_{1}\left(u_{c}\right)=\int_{0}^{1} u_{c}(t) \mathrm{d} t
$$

Here, we put $a=-2$ and by $u_{c}$ we denote a positive solution of (6.5) for a specific nonnegative value of $c$. In order to approximate $\left.\Phi_{1}\left(u_{c}\right)\right]$, we introduce a partition of the interval $[0,1]$ into equidistant subintervals of length $10^{-2}$. As a quadrature formula, we use the composed Gaussian rule with five evaluation points in each subinterval of $[0,1]$. The results can be found in Table 1 below.

\begin{tabular}{|r||c|}
\hline$c$ & $\Phi_{1}\left(u_{c}\right)$ \\
\hline \hline 0.0 & 0.259842454338672 \\
\hline 0.1 & 0.228105737635487 \\
\hline 0.2 & 0.235421595255397 \\
\hline 0.3 & 0.244242057208884 \\
\hline 0.5 & 0.264432168174144 \\
\hline 1.0 & 0.322297787747369 \\
\hline 2.0 & 0.521359670723535 \\
\hline 5.0 & 1.000102652113081 \\
\hline 10.0 & 1.819813323209159 \\
\hline 100.0 & 16.79012750689064 \\
\hline
\end{tabular}

Table 1: Problem (6.5).

Table 1 shows that $\Phi_{1}\left(u_{c}\right)$ is not monotonous for $c \in[0, \infty)$, that is the inequality $c_{1}<c_{2}$ need not imply $\Phi_{1}\left(u_{c_{1}}\right) \leq \Phi_{1}\left(u_{c_{2}}\right)$. But we know that there exists at least one $c_{*} \in[0, \infty)$ such that $\Phi_{1}\left(u_{c}\right)$ reaches its minimum at $u_{c^{*}}$.

\section{Acknowledgements}

This research was supported by the grant Matematické modely a struktury, PrF$2011-022$. 


\section{References}

[1] F. F. Abraham, Homogeneous Nucleation Theory, Acad. Press, New York 1974.

[2] R. P. Agarwal, M. Meehan, and D. O'Regan, Fixed Point Theory and Applications, Cambridge University Press, 2001.

[3] V. Bongiorno, L. E. Scriven, and H. T. Davis, Molecular theory of fluid interfaces, J. Colloid and Interface Science 57 (1967), 462-475.

[4] C. Budd, O. Koch, and E. Weinmüller, From nonlinear PDEs to singular ODEs, Appl. Num. Math. 56 (2006), 413-422.

[5] G. H. Derrick, Comments on nonlinear wave equations as models for elementary particles, J. Math. Physics 5 (1965), 1252-1254.

[6] P. C. Fife, Mathematical aspects of reacting and diffusing systems, Lecture notes in Biomathematics 28, Springer 1979.

[7] R. A. Fischer, The wave of advance of advantegeous genes, J. Eugenics 7 (1937), 355-369.

[8] H. Gouin and G. Rotoli, An analytical approximation of density profile and surface tension of microscopic bubbles for Van der Waals fluids, Mech. Research Communic. 24 (1997), 255-260.

[9] R. Hammerling, O. Koch, C. Simon, and E. Weinmüller, Numerical Solution of Eigenvalue Problems in Electronic Structure Computations, J. Comp. Phys. 181 (2010), 1557-1561.

[10] F. de Hoog, and R. Weiss, Difference methods for boundary value problems with a singularity of the first kind, SIAM J. Numer. Anal. 13 (1976), 775813.

[11] F. DE Hoog AND R. WeIss. Collocation methods for singular boundary value problems, SIAM J. Numer. Anal. 15 (1978), 198-217.

[12] G. Kitzhofer, O. Koch, P. Lima, and E. Weinmüller, Efficient Numerical Solution of the Density Profile Equation in Hydrodynamics, J. Sci. Comp. 32 (2007), 411-424.

[13] G. Kitzhofer, G. Pulverer, C. Simon, O. Koch, and E. Weinmüller, The New Matlab Solver BVPSUITE for the Solution of Singular Implicit BVPs, JNAIAM, J. Numer. Anal. Ind. Appl. Math. 5 (2010), 113-134. 
[14] N. B. Konyukhova, P. M. Lima, M. L. Morgado, and M. B. Sololviev, Bubbles and droplets in nonlinear physics models: analysis and numerical simulation of singular nonlinear boundary value problems, Comp. Maths. Math. Phys. 48 (2008), 2018-2058.

[15] A. P. Linde, Particle Physics and Inflationary Cosmology, Harwood Academic, Chur, Switzerland 1990.

[16] I. Rachůnková and S. Staněk, Properties of the set of positive solutions to Dirichlet boundary value problems with time singularities, Central European J. Math., to appear.

[17] I. Rachůnková, G. Pulverer, and E. Weinmüller, A unified approach to singular problems arising in the membrane theory, Applications of Mathematics 55 (2010), 47-75.

[18] J. D. van der Waals and R. Kohnstamm, Lehrbuch der Thermodynamik, Vol. 1, Leipzig 1908.

[19] E. B. Weinmüller, On the Boundary Value Problems of Ordinary Second Order Differential Equations with a Singularity of the First Kind, SIAM J. Math. Anal. 15 (1984), 287-307.

[20] E. B. Weinmüller, Collocation for singular boundary value problems of second order, SIAM J. Numer. Anal. 23 (1986), 1062-1095. 